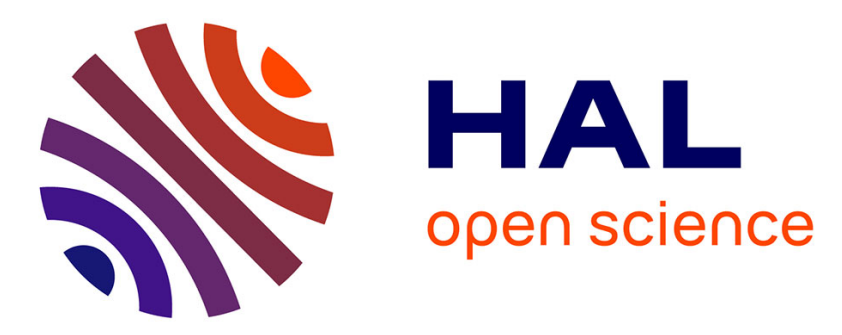

\title{
An upper bound for validity limits of asymptotic analytical approaches based on normal form theory
}

Claude-Henri Lamarque, Cyril Touzé, Olivier Thomas

\section{To cite this version:}

Claude-Henri Lamarque, Cyril Touzé, Olivier Thomas. An upper bound for validity limits of asymptotic analytical approaches based on normal form theory. Nonlinear Dynamics, 2012, 70 (3), pp.19311949. 10.1007/s11071-012-0584-y . hal-00880968

\section{HAL Id: hal-00880968 https://hal.science/hal-00880968}

Submitted on 5 Dec 2013

HAL is a multi-disciplinary open access archive for the deposit and dissemination of scientific research documents, whether they are published or not. The documents may come from teaching and research institutions in France or abroad, or from public or private research centers.
L'archive ouverte pluridisciplinaire HAL, est destinée au dépôt et à la diffusion de documents scientifiques de niveau recherche, publiés ou non, émanant des établissements d'enseignement et de recherche français ou étrangers, des laboratoires publics ou privés. 


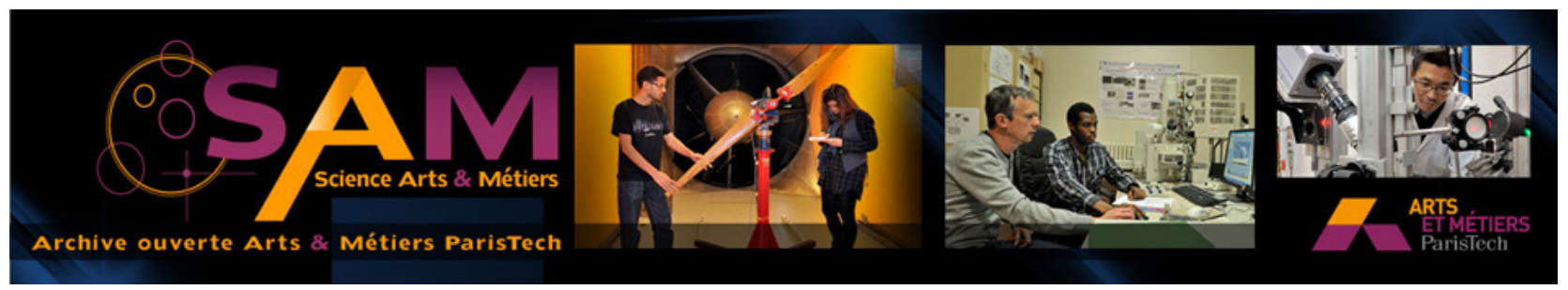

Science Arts \& Métiers (SAM)

is an open access repository that collects the work of Arts et Métiers ParisTech researchers and makes it freely available over the web where possible.

This is an author-deposited version published in: http://sam.ensam.eu

Handle ID: .http://hdl.handle.net/10985/7473

\section{To cite this version :}

Claude-Henri LAMARQUE, Cyril TOUZÉ, Olivier THOMAS - An upper bound for validity limits of asymptotic analytical approaches based on normal form theory - Nonlinear Dynamics - Vol. 70, n`3, p.1931-1949- 2012 


\title{
An upper bound for validity limits of asymptotic analytical approaches based on normal form theory
}

\author{
Claude-Henri Lamarque • Cyril Touzé • \\ Olivier Thomas
}

\begin{abstract}
Perturbation methods are routinely used in all fields of applied mathematics where analytical solutions for nonlinear dynamical systems are searched. Among them, normal form theory provides a reliable method for systematically simplifying dynamical systems via nonlinear change of coordinates, and is also used in a mechanical context to define Nonlinear Normal Modes (NNMs). The main recognized drawback of perturbation methods is the absence of a criterion establishing their range of validity in terms of amplitude. In this paper, we propose a method to obtain upper bounds for amplitudes of changes of variables in normal form transformations. The criterion is tested on simple mechanical systems with one and two degrees-of-freedom, and for complex as well as real normal form. Its behavior with increasing order in the normal transform is established, and comparisons are drawn between exact solutions and normal form com-
\end{abstract}

\section{C.-H. Lamarque}

Université de Lyon, ENTPE/DGCB/FRE CNRS 3237,

Rue Maurice Audin, 69518, Vaulx-en-Velin Cedex, France

e-mail: lamarque@entpe.fr

C. Touzé $(\bowtie)$

Unité de Mécanique (UME), ENSTA—ParisTech, Paris, France

e-mail: cyril.touze@ensta-paristech.fr

O. Thomas

Structural Mechanics and Coupled Systems Laboratory, CNAM, Paris, France

e-mail: olivier.thomas@cnam.fr putations for increasing levels of amplitudes. The results clearly establish that the criterion gives an upper bound for validity limit of normal transforms.

Keywords Normal form theory - Upper bound of validity limit · Perturbation methods · Nonlinear Normal Modes

\section{Introduction}

Perturbation methods allow deriving analytical solutions of nonlinear dynamical systems by assuming that the solution will be a perturbation of the linear one that can be analytically derived by ad-hoc hypotheses and application of the Fredholm alternative. The very first premises of analytical works involving a perturbative scheme dates back to the eighteenth century. The main developments have then been pushed forward from the end of the nineteenth century and the pioneering works of Poincaré, Lindstedt, Birkhoff and Lyapunov, among others, for solving difficult problems in celestial mechanics like the three-body problem [1-3]. They are now widely used in all fields of applied mathematics and have been very successful in providing a large amount of predictive results. In a mechanical context, they are used to derive approximate expressions for free and forced responses of nonlinear (smooth) mechanical systems with various kinds of nonlinearities. The books by Nayfeh et al., provide a nice and complete picture of all the results that can be expected 
from such methods $[4,5]$. Successive approximations, generally ordered by a perturbative small parameter $\varepsilon$, are found by analytical developments that get more and more involved with the required perturbation order, so that practically, calculations are limited to inclusion of first- or second-order of perturbation.

Normal form theory, which can be viewed as a perturbation method [6], lays down on the fundamental theorems of Poincaré and Poincaré-Dulac $[1,7]$. It is a general and powerful method that allows simplification of nonlinear terms of a dynamical system, by distinguishing resonant and nonresonant terms, so that eventually one is able to derive the "skeleton" of the dynamical system containing only the important terms for dynamical behaviors that are responsible for bifurcations and the nature of solutions, in the vicinity of a special solution such as fixed points or periodic orbits [8-10, 16, 17, 21-24]. In the mechanical context, normal form can be used to derive several important analytical results, as well as for showing equivalences with other methods such as NNMs, or appearance of small denominators in perturbative schemes, hence making it a cornerstone of all perturbation techniques $[14,15,25,26]$. The computation technique for deriving normal forms and coefficients of the associated nonlinear change of coordinates can be efficiently automatized by using symbolic computational toolboxes, as shown, for example, in [18, 19]. Recently, it has also been applied to the second-order vibration problem [20], in a manner sharing many common points with the real normal form method developed in $[25,26]$.

The recognized drawback of all the perturbation methods that is also shared by normal form theory, is the absence of a criterion establishing the range of validity of the approximate solutions, in terms of amplitudes of the coordinates. Even though one expects the radius of convergence of the approximate solution to increase with the order of the development used for the perturbation method employed, no validity limits have ever been given, so that one is led to apply direct numerical integration of the original dynamical system in order to assess the validity range of the approximate solution.

In this paper, an upper bound for the validity limit of asymptotic expansions based on normal form theory, is exhibited. The key relies in interpreting the homological equations that has to be solved in the normal form process in order to derive the coefficients of the nonlinear change of coordinates, as an inver- sion procedure that is generally not performed in classical calculations. The criterion is presented in Sect. 2, after recalling the ground basis of normal form theory. The method is then applied to a single degree-offreedom (dof) Duffing equation with cubic nonlinearity in Sect. 3, where classical complex normal form transformations are used. For the single dof Duffing equation, normal forms up to a high order are computable so that the convergence of the bound with increasing order can be studied. A two dofs system with real normal form procedure is then considered in Sect. 4, where the case of adding linear viscous damping terms in the mechanical system is also highlighted. Extensions of the criterion to N-dofs systems and the comparison with NNMs is also discussed. Finally, conclusions are drawn in Sect. 5.

\section{Classical normal form theory}

In this section, we recall the basic normal form theory for an autonomous finite dimensional nonlinear dynamical system written as

$\dot{x}=f(x)=L_{0} x+g(x)$

where $\boldsymbol{x}$ is a $n$ dimensional vector, $\boldsymbol{x} \in \mathcal{E}, \mathcal{E}=\mathbb{R}^{n}$ or $\mathbb{C}^{n}$ is the phase space, and $\dot{\boldsymbol{x}}=\mathrm{d} \boldsymbol{x} / \mathrm{d} t$, with $t$ the time. $\boldsymbol{f}: \mathcal{E} \longrightarrow \mathcal{E}$ is a $n$-dimensional vector field, $\boldsymbol{L}_{0}$ denotes $\partial \boldsymbol{f}(\mathbf{0})$ the Jacobian matrix of $\boldsymbol{f}$ at $\boldsymbol{x}=\mathbf{0}$, and $g$ stands for the higher-order terms (at least order 2) of $f$.

We assume that $\boldsymbol{f}$ is given by a power series expansion (a finite sum or an analytical function), and that the origin of phase space is a fixed point $(\boldsymbol{f}(\mathbf{0})=\mathbf{0})$. This frame permits us to deal with usual cases where $\boldsymbol{L}_{0}$ is a real matrix or a complex matrix (e.g., in diagonal form).

\subsection{Normal form}

The idea of the normal form theory is to simplify as much as possible Eq. (1) by means of a nonlinear change of coordinates. The principle is as follows: Let us give an order $k$; let us determine polynomials $\boldsymbol{\Phi}$ and $\boldsymbol{R}$ of degree $\leq k(\boldsymbol{\Phi}, \boldsymbol{R}: \mathcal{E} \longrightarrow \mathcal{E})$ such as $\boldsymbol{\Phi}(\mathbf{0})=\boldsymbol{R}(\mathbf{0})=\mathbf{0}$ and $\partial \boldsymbol{\Phi}(\mathbf{0})=\partial \boldsymbol{R}(\mathbf{0})=\mathbf{0}$ and $\boldsymbol{R}$ is as simple as possible (if possible $\mathbf{0}$ ), and so that the change of variables:

$\boldsymbol{x}=\boldsymbol{u}+\boldsymbol{\Phi}(\boldsymbol{u})$ 
transforms (1) into

$\dot{\boldsymbol{u}}=\boldsymbol{L}_{0} \boldsymbol{u}+\boldsymbol{R}(\boldsymbol{u})+o\left(\|\boldsymbol{u}\|^{k}\right)$,

where the number of terms in $\boldsymbol{R}$ is very small as compared to $\boldsymbol{g}$ in (1). The $n$-dimensional vector $\boldsymbol{u}(\boldsymbol{u} \in \mathcal{E})$ denotes the new variable and the dynamical system (3) is called the normal form of the original system (1).

Let us denote $\left\{\lambda_{i}\right\}_{i=1, \ldots, n}$ the eigenvalues of $\boldsymbol{L}_{0}$. The most simple case is given by Poincaré's theorem [1], which states that as long as no resonance exists between the eigenvalues of $\boldsymbol{L}_{0}$, then it is possible to find $\boldsymbol{\Phi}$ such that $\boldsymbol{R}$ is zero. Resonances in the spectrum for order $k$ are defined by relationships of the form:

$$
\begin{aligned}
& \exists s=1, \ldots, n \\
& \lambda_{s}=\sum_{i=1}^{n} m_{i} \lambda_{i}, \quad m_{i} \geq 0, \quad \sum_{i=1}^{n} m_{i}=k \geq 2 .
\end{aligned}
$$

When resonances are present, Poincaré-Dulac's theorem states that all nonresonant terms can be cancelled so that $\boldsymbol{R}$ is only composed of resonant monomial terms [7]. For applications to mechanical conservative vibratory systems, resonant terms are still present due to the fact that the spectrum is composed of pairs of complex conjugate eigenvalues, so that $\boldsymbol{R}$ is not reduced to the zero vector.

\subsection{Homological equation}

In the normal form transformation, the unknown is the introduced function $\boldsymbol{\Phi}$. Poincaré's theorem provides a constructive method to compute $\boldsymbol{\Phi}$ by increasing orders of nonlinearity, through a so-called homological equation. Let us write Taylor expansion of all the terms: we note, for $\boldsymbol{f}, \boldsymbol{\Phi}$, and $\boldsymbol{R}$ :

$$
\begin{aligned}
& \boldsymbol{f}(\boldsymbol{u})=\boldsymbol{L}_{0} \boldsymbol{u}+\sum_{i=2}^{k} \boldsymbol{f}^{(i)}(\boldsymbol{u})+o\left(\|\boldsymbol{u}\|^{k}\right), \\
& \boldsymbol{\Phi}(\boldsymbol{u})=\sum_{i=2}^{k} \boldsymbol{\Phi}^{(i)}(\boldsymbol{u}), \\
& \boldsymbol{R}(\boldsymbol{u})=\sum_{i=2}^{k} \boldsymbol{R}^{(i)}(\boldsymbol{u}),
\end{aligned}
$$

where all the indexes $i$ denote the total degree of the corresponding polynomials, e.g., $\boldsymbol{f}^{(i)}$ denotes a polynomial of degree $i$ in variables $\boldsymbol{u}=\left(u_{1}, \ldots, u_{n}\right)^{\mathrm{T}}$. Let us replace in Eq. (1):

$$
\frac{\mathrm{d}}{\mathrm{d} t}(\boldsymbol{u}+\boldsymbol{\Phi}(\boldsymbol{u}))=(\boldsymbol{I}+\partial \boldsymbol{\Phi}) \dot{\boldsymbol{u}}=\boldsymbol{f}(\boldsymbol{u}+\boldsymbol{\Phi}(\boldsymbol{u})),
$$

where $I$ denotes the identity operator. Then, since the final result (Eq. (3)) gives $\dot{\boldsymbol{u}}=\boldsymbol{L}_{0} \boldsymbol{u}+\boldsymbol{R}(\boldsymbol{u})$, we obtain

$(\boldsymbol{I}+\partial \boldsymbol{\Phi})\left(\boldsymbol{L}_{0} \boldsymbol{u}+\boldsymbol{R}(\boldsymbol{u})\right)=\boldsymbol{f}(\boldsymbol{u}+\boldsymbol{\Phi}(\boldsymbol{u}))$.

This equality is expanded and ordered from degree 1 to degree $k$ :

- at degree 1, we check $\boldsymbol{L}_{0} \boldsymbol{u}=\boldsymbol{L}_{0} \boldsymbol{u}$

- then for degree $i,(2 \leq i \leq k)$, we obtain

$$
\boldsymbol{L}_{0} \Phi^{(i)}(\boldsymbol{u})-\partial \boldsymbol{\Phi}^{(i)}(\boldsymbol{u}) \boldsymbol{L}_{0} \boldsymbol{u}=\boldsymbol{R}^{(i)}(\boldsymbol{u})-\boldsymbol{P}^{(i)}(\boldsymbol{u}),
$$

where $\boldsymbol{P}^{(i)}$ is a polynomial of degree $i$ in $\boldsymbol{u}$, that depends only on: ${ }^{1}$

- $\boldsymbol{f}^{(j)}, 2 \leq j \leq i$

- $\boldsymbol{\Phi}^{(j)}, 2 \leq j \leq i-1$

- $\boldsymbol{R}^{(j)}, 2 \leq j \leq i-1$

We obtain a triangular system of equations for the unknowns $\boldsymbol{\Phi}^{(j)}$ that can be written on a basis leading to solving linear systems $[8,11]$. The Fredholm alternative provides both the resonant terms $\boldsymbol{R}^{(j)}(2 \leq j \leq$ $k)$ and the normal transforms $\boldsymbol{\Phi}^{(j)}(2 \leq j \leq k)$. However, the latter one is not uniquely defined, as the typical structure of the problem (in case of diagonal operator $\left.\boldsymbol{L}_{0}\right)$ is as follows. For the solvable part of the system (nonresonant terms), equations for $\boldsymbol{\Phi}^{(j)}$ appears to be of the form:

$a \neq 0, \quad a \Phi=b \Rightarrow \Phi=b / a$,

where $\Phi$ denotes one of the coefficients of the normal transform $\boldsymbol{\Phi}^{(j)}$. This leads us finally, for each order $j$, to a unique solution for $\boldsymbol{\Phi}^{(j)}$. This is not the case for the singular parts of the linear system (resonant terms), where application of the Fredholm alternative lends us with problems of the form:

$0 \cdot \Phi=R-P$,

\footnotetext{
${ }^{1}$ Precisely, following Eq. (9), one obtains: $\boldsymbol{P}^{(i)}=\left[\boldsymbol{f}^{(i)}(\boldsymbol{u}+\right.$ $\boldsymbol{\Phi}(\boldsymbol{u}))]^{(i)}-[\partial \boldsymbol{\Phi} \boldsymbol{R}(\boldsymbol{u})]^{(i)}$ where $[\circ]^{(i)}$ denotes all the monomial of degree $i$ of $\mathrm{o}$.
} 
where $R$ and $P$ denote the coefficients of respectively $\boldsymbol{R}^{(j)}$ and $\boldsymbol{P}^{(j)}$. In Eq. (12), the choice for $\Phi$ is not unique. The most common choice retained in literature is to let $\Phi=0$, hence leading to the simplest expression of normal transform, as the resonant terms staying in the normal form after nonlinear change of coordinates are simply given by $R=P$. However, one must keep in mind that any other choice can be made.

At the end of the process, for each order of nonlinearity $k$, one obtains the change of coordinates (2) and the normal form (3) (the reduced equations). Normal forms can then be used in bifurcation theory to classify the generic families of bifurcations in dynamical systems $[8,11]$. For mechanical vibratory systems, it can also be used to define Nonlinear Normal Modes (NNMs) and build reduced-order models [14, 25, 26]. However, one recognized drawback of the method is that the validity limit of the change of variables is not given. Hence, as $\boldsymbol{u}$ is getting large, one has to resort to numerical verification to verify that the approximate solution is still valid. In the next section, we propose an upper bound for this validity limit.

\subsection{Practical convergence}

Normal form calculations are made in practice up to a given finite degree $k$. So only $\boldsymbol{R}^{(2)}, \ldots, \boldsymbol{R}^{(k)}$, $\boldsymbol{\Phi}^{(2)}, \ldots, \boldsymbol{\Phi}^{(k)}$ up to degree $k$ are computed. Let us note that in fact Eq. (8) has to be understood as:

$\dot{\boldsymbol{u}}=(\boldsymbol{I}+\partial \boldsymbol{\Phi}(\boldsymbol{u}))^{-1} \boldsymbol{f}(\boldsymbol{u}+\Phi(\boldsymbol{u}))$,

leading to $\dot{\boldsymbol{u}}=\boldsymbol{L}_{0} \boldsymbol{u}+\boldsymbol{R}(\boldsymbol{u})$. Even though Eq. (13) is never written when one computes its normal transform degree by degree (as in general one solves Eq. (9) without thinking of inversing the left-hand side term), the problem can be treated similarly by inverting $\boldsymbol{I}+\partial \boldsymbol{\Phi}(\boldsymbol{u})$. This remark gives us the key to set the upper bound for validity limit. For practical use of normal transform, we propose to set a boundary associated to the distance of $\boldsymbol{u}$ from $\mathbf{0} \in \mathcal{E}$, so that $\boldsymbol{I}+\partial \boldsymbol{\Phi}(\boldsymbol{u})$ becomes singular and $(\boldsymbol{I}+\partial \boldsymbol{\Phi}(\boldsymbol{u}))^{-1} \boldsymbol{f}(\boldsymbol{u}+\boldsymbol{\Phi}(\boldsymbol{u}))$ keeps also a singular point (it means the limit of the term $\boldsymbol{f}(\boldsymbol{u}+\boldsymbol{\Phi}(\boldsymbol{u}))$ when $\boldsymbol{u}$ tends to singular value of $(\boldsymbol{I}+\partial \boldsymbol{\Phi}(\boldsymbol{u}))$ does not suppress the singularity).

Since in approximated problems we compute only the terms $\boldsymbol{\Phi}^{(2)}, \ldots, \boldsymbol{\Phi}^{(k)}$ and since we organize nonlinearities according to increasing degrees in $\boldsymbol{f}(\boldsymbol{u}+$ $\boldsymbol{\Phi}(\boldsymbol{u})$ ), we propose in practice to look for $\boldsymbol{u}$ such that:

$$
\begin{aligned}
\Delta(\boldsymbol{u}) & =\operatorname{det}\left(\boldsymbol{I}+\partial \boldsymbol{\Phi}^{(2)}(\boldsymbol{u})+\cdots+\partial \boldsymbol{\Phi}^{(k)}(\boldsymbol{u})\right) \\
& =0
\end{aligned}
$$

and such that

$\lim _{\boldsymbol{v} \longrightarrow \boldsymbol{u}, \Delta(\boldsymbol{u})=0}\left|\frac{T\left[\operatorname{adj}(\boldsymbol{I}+\partial \boldsymbol{\Phi}(\boldsymbol{v})) \boldsymbol{f}\left(\boldsymbol{v}+\boldsymbol{\Phi}^{(2)}(\boldsymbol{v})+\cdots+\boldsymbol{\Phi}^{(k)}(\boldsymbol{v})\right)\right]}{\Delta(\boldsymbol{v})}\right|=+\infty$,

where $\operatorname{adj}(\bullet)$ is the transpose of the cofactor matrix of - and $T[\bullet]$ is the truncated Taylor expansion of $\bullet$ up to order $n(k-1)$.

The remainder of the paper is devoted to assessing the accuracy of the proposed criterion for validity limit, for various, simple mechanical systems. We begin with the Duffing equation, for which normal transformations are computable up to a high order $k$ (at least for free oscillations). This will allow verification of convergence of the proposed criterion with respect to perturbation order. Note that throughout the paper, cases of internal resonance between the eigenfrequencies of the vibratory system are not taken into account, so that the simplest normal transform as shown, for example, in [14, 25, 26], is considered.

\section{Duffing equation with complex normal transform}

Let us consider the following Duffing equation, a usual second-order problem corresponding to a one degreeof-freedom mechanical system:

$\ddot{w}_{1}+\omega_{0}^{2} w_{1}+c w_{1}^{3}=0$,

where $\omega_{0}$ and $c$ are real parameters and $w_{1}(t)$ is the unknown time function. 
3.1 Normal form calculation with simplest form of normal transform

Let us give normal form calculations up to order 3. Let us first transform (16) in diagonal form, by introducing the linear change of variables

$\dot{w}_{1}=\lambda_{1} x_{1}+\lambda_{2} x_{2}, \quad w_{1}=x_{1}+x_{2}$,

where $\lambda_{1}=\overline{\lambda_{2}}=-i \omega_{0}$ are the eigenvalues of the linear part of (16) rewritten in a first-order form and $i^{2}=-1$. Equation (16) is then transformed into

$$
\begin{aligned}
& \dot{x}_{1}=\lambda_{1} x_{1}+\frac{i c}{2 \omega_{0}}\left(x_{1}+x_{2}\right)^{3}, \\
& \dot{x}_{2}=\lambda_{2} x_{2}-\frac{i c}{2 \omega_{0}}\left(x_{1}+x_{2}\right)^{3},
\end{aligned}
$$

which are two complex conjugate differential equations on the unknowns $x_{1}$ and $x_{2}$ and which define vector field $\boldsymbol{f}$ and matrix $\boldsymbol{L}_{0}=\operatorname{diag}\left(\lambda_{1}, \lambda_{2}\right)$.

Normal form up to order 3 is then calculated for this two-dimensional differential system of order 1 by setting

$$
x_{1}=u_{1}+\Phi_{1}\left(u_{1}, u_{2}\right), \quad x_{2}=u_{2}+\Phi_{2}\left(u_{1}, u_{2}\right),
$$

where

$\Phi_{1}\left(u_{1}, u_{2}\right)=-\frac{1}{8} \frac{c u_{2}^{3}}{\omega_{0}^{2}}-\frac{3}{4} \frac{c u_{1} u_{2}^{2}}{\omega_{0}^{2}}+\frac{1}{4} \frac{c u_{1}^{3}}{\omega_{0}^{2}}$,

$\Phi_{2}$ conjugate of $\Phi_{1}$.

Normal form up to order 3 is then

$$
\begin{aligned}
& \dot{u}_{1}=-i \omega_{0} u_{1}-\frac{3 i c}{2 \omega_{0}} u_{1}^{2} u_{2}, \\
& \dot{u}_{2}=i \omega_{0} u_{2}+\frac{3 i c}{2 \omega_{0}} u_{2}^{2} u_{1},
\end{aligned}
$$

where two resonant terms have to be kept.

Following the procedure exposed in Sect. 2.3, the determinant of $\boldsymbol{I}+\partial \boldsymbol{\Phi}(\boldsymbol{u})$, with $\boldsymbol{u}=\left(\begin{array}{ll}u_{1} & u_{2}\end{array}\right)^{\mathrm{T}}$ and $\boldsymbol{\Phi}=\left(\Phi_{1} \Phi_{2}\right)^{\mathrm{T}}$, is equal to

$\Delta(\boldsymbol{u})=-\frac{\left(6 c u_{2}^{2}+3 c u_{1} u_{2}+6 c u_{1}^{2}-8 \omega_{0}^{2}\right)\left(6 c u_{2}^{2}+3 c u_{1} u_{2}+6 c u_{1}^{2}+8 \omega_{0}^{2}\right)}{64 \omega_{0}{ }^{4}}$

Since $u_{1}$ and $u_{2}$ are complex conjugate, we define $P$ and $Q$ to be two real variables so that $u_{1}=P+i Q$. By rewriting both factors of the numerator of $\Delta(\boldsymbol{u})$ as functions of $P$ and $Q$, one can show that the matrix $\boldsymbol{I}+\partial \boldsymbol{\Phi}(\boldsymbol{u})$ is singular $(\Delta(\boldsymbol{u})=0)$ if

$$
\begin{aligned}
& 15 c P^{2}-9 c Q^{2}-8 \omega_{0}^{2}=0 \text { or } \\
& 15 c P^{2}-9 c Q^{2}+8 \omega_{0}^{2}=0,
\end{aligned}
$$

which defines two curves in the $(P, Q)$ plane, shown on Fig. 1(a).

Similar calculations have been done with the help of symbolic computations for higher order normal forms and simplest choice of the normal transform (the component of the normal transform according to each resonant term in homological equation is cancelled as in Eq. (12)). Details for normal transforms and normal forms are omitted here for sake of brevity (except degree 11 which is given in Appendix A for illustrating the increasing complexity with respect to the selected order $k)$. We simply provide in the plane $(P, Q)$ the corresponding results to show that there is a limit ball centered on $\boldsymbol{u}=0$ so that $\boldsymbol{I}+\partial \Phi(\boldsymbol{u})$ is not singular.

The maximal convergence radius (i.e., the maximal modulus $\left|u_{1}\right|=\left|u_{2}\right|$ of $u_{1}$ and $u_{2}$ before the circle centered on $P=Q=0$ meets the curve of singularities) corresponding to the successive orders $k$ of normal form calculations are given in Table 1 . From this result, it is reasonable to claim that upper bound of convergence radius tends to $1 / 2$. This corresponds to a limit value which sounds reasonable for a perturbation approach. Going back to the original variable $w_{1}$ in Eq. (16), since $w_{1}=u_{1}+u_{2}=2 P$, it is meaningful to demand $\left|w_{1}\right| \leq 1$. Note that when $c<0$, two saddles appears with values $\left(w_{1}, \dot{w}_{1}\right)=( \pm 1 / \sqrt{-c}, 0)$. Hence, when $c \in[-1,-\infty$ [, the upper bound may be smaller due to the existence of limit cycles that are limited in phase space by the presence of these two fixed points. 
Fig. 1 Singularity curves in the plane $(P, Q)$, where the normal coordinate $u_{1}=P+i Q$, for $\omega_{0}=1$, $c=1$ and the normal form calculation up to order: 3 (a), 5 (b), 7 (c), 11 (d), 17 (e), 25 (f). A circle of radius 0.5 is shown by the dotted line (a) - Order 3

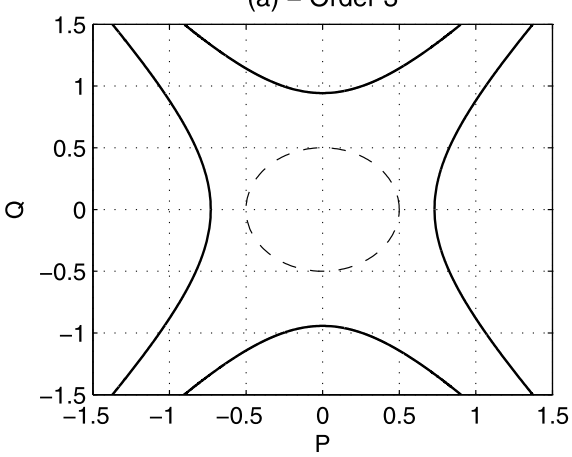

(c) - Order 7

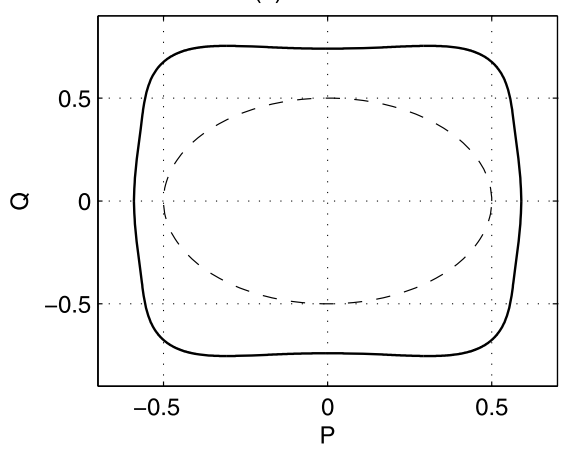

(e) - Order 17

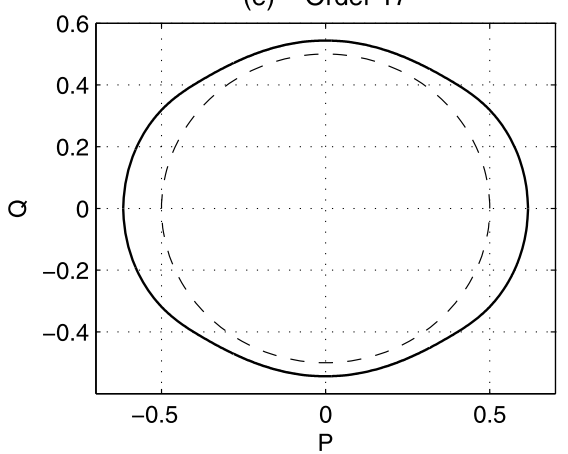

(b) - Order 5

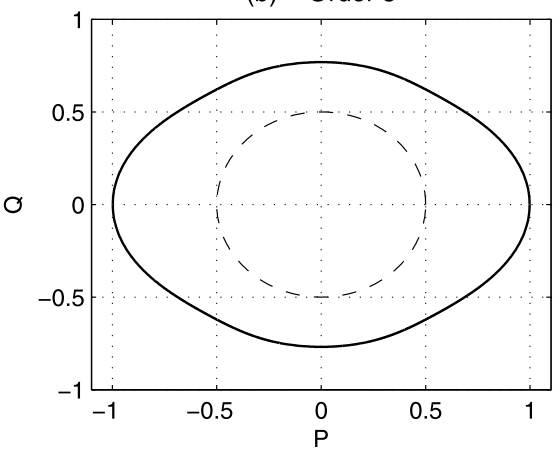

(d) - Order 11
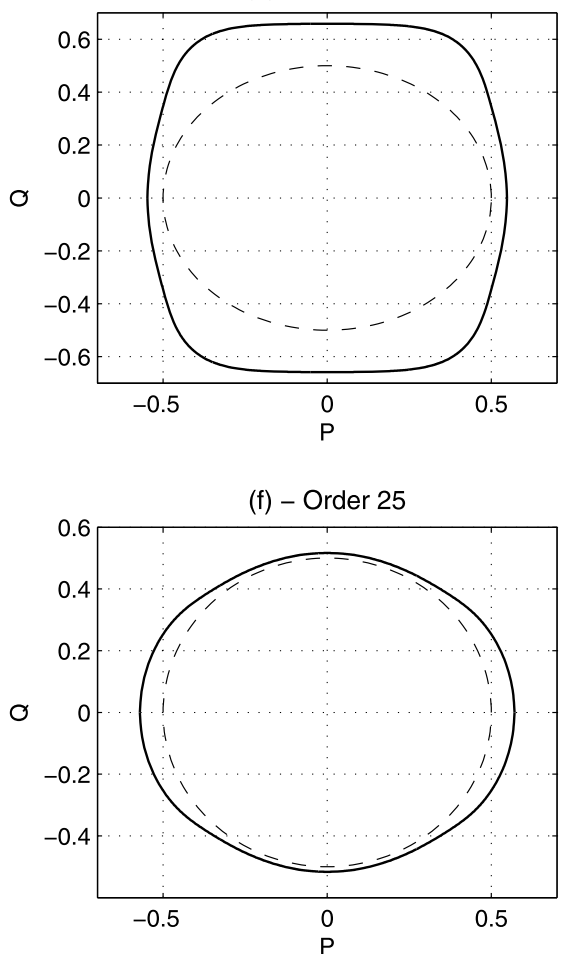

We also checked that the polynomial corresponding to $\Delta(\boldsymbol{u})$ does not divide the first component of the term corresponding to

$T\left[\operatorname{adj}(\boldsymbol{I}+\partial \boldsymbol{\Phi}(\boldsymbol{v})) \boldsymbol{f}\left(\boldsymbol{v}+\boldsymbol{\Phi}^{(2)}(\boldsymbol{v})+\cdots+\boldsymbol{\Phi}^{(k)}(\boldsymbol{v})\right)\right]$, (up to coherent order: 4 here) to verify Eq. (15). For a normal form at order 3, the ratio associated with (15), up to the normal form calculations of order 3 , is

$$
\frac{32 i u_{1}\left(2 \omega_{0}^{2}+3 c u_{1} u_{2}\right) \omega_{0}^{3}}{\left(6 c u_{2}^{2}+3 c u_{1} u_{2}+6 c u_{1}^{2}-8 \omega_{0}^{2}\right)\left(6 c u_{2}^{2}+3 c u_{1} u_{2}+6 c u_{1}^{2}+8 \omega_{0}^{2}\right)}
$$


Table 1 Maximal convergence radius (i.e., maximal modulus of $u_{1}$ and $\left.u_{2}\left(\left|u_{1}\right|=\left|u_{2}\right|\right)\right)$ as a function of order $k$ of normal form calculation

\begin{tabular}{ll}
\hline Normal form order $\mathrm{k}$ & Maximal modulus of $u_{1}$ \\
\hline 3 & 0.7303 \\
5 & 0.7688 \\
7 & 0.5898 \\
11 & 0.5473 \\
17 & 0.5442 \\
25 & 0.5171 \\
27 & 0.4981 \\
\hline
\end{tabular}

The above equation can be rewritten in term of $P$ and $Q$, so that zeros of its denominator verify Eqs. (23) and its numerator corresponds to

$32 i \omega_{0}^{3}(P+i Q)\left(2 \omega_{0}^{2}+3 c\left(P^{2}+Q^{2}\right)\right)$.

The limit of this numerator, when the first or the second of Eqs. (23) is verified, is different from 0, so that the singularities of $\boldsymbol{I}+\partial \boldsymbol{\Phi}(\boldsymbol{u})$ cannot be compensated by eventual singularities of $\boldsymbol{f}(\boldsymbol{u}+\boldsymbol{\Phi}(\boldsymbol{u}))$.

\subsection{Normal form calculation with another normal transform: a simple example}

We recall in Sect. 2.2 that the normal transform is not unique. The choice of $\mathbf{0}$ as the vector of the kernel in the Fredholm alternative (i.e., $\Phi=0$ in Eq. (12)) corresponds to the simplest normal transform. Since another choice can be made ( $\Phi \neq 0$ in Eq. (12)), is it possible to suppress the singular points of $\boldsymbol{I}+\partial \boldsymbol{\Phi}(\boldsymbol{u})$ via this different choice?

The general case seems to be an open question even if intuitively we may think that the choice of such a nonzero term will not modify the geometry of the problem and so singular points will persist. To illustrate the question, let us consider normal transform for the simple one degree-of-freedom system of Sect. 3.1. As we will see, with calculations up to order 3 , it is impossible to suppress the singular points.

The most general expression of normal transform up to order 3 for (18) corresponds to

$$
\begin{aligned}
& \Phi_{1}^{*}\left(u_{1}, u_{2}\right)=\Phi_{1}\left(u_{1}, u_{2}\right)+\beta u_{1}^{2} u_{2}, \\
& \Phi_{2}^{*} \text { conjugate of } \Phi_{1}^{*},
\end{aligned}
$$

where $\Phi_{1}\left(u_{1}, u_{2}\right)$ is given by Eq. (20), $\beta$ is a given complex coefficient, and $u_{1}$ and $u_{2}$ are conjugate. The normal form up to order 3 is not modified and is given by (21).

Now, determinant of $\boldsymbol{I}+\partial \boldsymbol{\Phi}(\boldsymbol{u})$ is found to be

$$
\begin{aligned}
\Delta(\boldsymbol{u})= & 1+2(\beta+\bar{\beta}) u_{1} u_{2}+\frac{3}{8}\left(\beta-\frac{3}{2}\right) u_{1}^{4} \\
& +\frac{3}{8}\left(\bar{\beta}-\frac{3}{2}\right) u_{2}^{4}+3\left(\beta \bar{\beta}-\frac{27}{64}\right) u_{1}^{2} u_{2}^{2} \\
& +\frac{3}{2} \bar{\beta} u_{1}^{3} u_{2}+\frac{3}{2} \beta u_{1} u_{2}^{3}-\frac{9}{16} u_{2}^{3} u_{1} \\
& -\frac{9}{16} u_{1}^{3} u_{2} .
\end{aligned}
$$

Introducing $u_{1}=P+i Q, u_{2}=P-i Q, \beta=r+i t$ and $P=s Q$ with $P, Q, r, s$, and $t$ real numbers, one has to study the behavior of the following polynomial in $Q^{2}$ versus coefficients $(r, s, t)$ :

$$
\begin{aligned}
1+ & {\left[4 r+4 r s^{2}\right] Q^{2}+\left[3 s^{4} t^{2}+6 s^{2} t^{2}-\frac{9}{4} r+3 t^{2}\right.} \\
& +3 r^{2}+\frac{15}{4} s^{4} r+9 s t+\cdots+3 s^{3} t-\frac{9}{2} r s^{2}-\frac{81}{64} \\
& \left.+\frac{135}{32} s^{2}-\frac{225}{64} s^{4}+6 s^{2} r^{2}+3 s^{4} r^{2}\right] Q^{4} .
\end{aligned}
$$

Indeed, one has to examine if it is possible to chose $r, t$ so that this polynomial has no roots for any given $s$. Cumbersome analytical calculations proves that this is impossible (see Appendix B).

Another question is associated with the choice of a nonzero $\beta$ : Is it possible to increase the radius of convergence? In other words, could we increase the value of $\left|u_{1}\right|=\left|u_{2}\right|$ where a singular point of $\boldsymbol{I}+\partial \boldsymbol{\Phi}(\boldsymbol{u})$ occurs? Obtaining an answer to this question is difficult through an analytical method. However, numerical investigations show that in the present case of an order 3 normal form, it is impossible to increase the radius.

\section{A two-dofs system with real normal form}

In this section, the upper bound is derived on a two dofs system displaying quadratic and cubic nonlinearity. Computations are realized through a real formulation, up to third order, of the normal transformation. The validity limit provided by the proposed cri- 
terion of Sect. 2.3 is compared to dynamical data obtained by comparing exact solutions with third-order asymptotics. The effect of the damping is also studied. Finally, a comparison is drawn with nonlinear normal modes (NNMs) calculations based on the center manifold technique, and a simplified criterion is discussed.

\subsection{Real normal form}

Real normal form computations have been derived for mechanical systems, where the linear part is of the oscillator-type, and thus contains a second-derivative with respect to time. The idea of using a real transformation is that, even though the system is reduced to its first-order form during the computations, second-order formulations are recovered at the end of the process so that one has always oscillator equations at hand. The general transformation has been derived for undamped vibratory systems in [25] and for damped systems in [26]. The main steps are here briefly recalled for the sake of completeness.

The starting point is an assembly of $N$ nonlinear oscillators displaying quadratic and cubic nonlinearity. It is assumed that the linear part has been made diagonal by use of the eigenmodes of the system, so that the dynamics reads, $\forall p=1, \ldots, N$ :

$$
\begin{gathered}
\ddot{X}_{p}+\omega_{p}^{2} X_{p}+\sum_{i=1}^{+\infty} \sum_{j \geq i}^{+\infty} g_{i j}^{p} X_{i} X_{j} \\
+\sum_{i=1}^{+\infty} \sum_{j \geq i}^{+\infty} \sum_{k \geq j}^{+\infty} h_{i j k}^{p} X_{i} X_{j} X_{k}=0 .
\end{gathered}
$$

A first-order formulation of (28) is used by introducing the velocities $Y_{p}=\dot{X}_{p}$ as complementary variables so as to fit the general framework explained in Sect. 2, Eq. (1). Hence, the vector of generalized coordinates writes $\boldsymbol{x}=\left(X_{1} Y_{1} \cdots X_{N} Y_{N}\right)^{\mathrm{T}}$ and the matrix $\boldsymbol{L}_{0}$ is $2 N \times 2 N$ and composed of $N$ diagonal blocks of the form:

$$
\left(\begin{array}{cc}
0 & 1 \\
-\omega_{p}^{2} & 0
\end{array}\right) .
$$

The nonlinear coordinate change is of the general form given by (2): $\boldsymbol{x}=\boldsymbol{u}+\boldsymbol{\Phi}(\boldsymbol{u})$, where $\boldsymbol{u}$ is a vector of newly introduced variables, and reads: $\boldsymbol{u}=$
$\left(R_{1} S_{1} \cdots R_{N} S_{N}\right)^{\mathrm{T}}$. Pairs of coordinates $\left(R_{p}, S_{p}\right)$ are respectively homogeneous to a displacement and a velocity; they represent the motions in a curved grid spanned by the invariant manifolds and are sometimes called normal coordinates [25, 26]. The nonlinear transformation $\boldsymbol{\Phi}(\boldsymbol{u})$ is computed by successive elimination of non-resonant terms. Calculations are realized up to order three in $[25,26]$ so that polynomial asymptotic expansions are obtained in the form, $\forall p=1, \ldots, N$ :

$$
\begin{gathered}
X_{p}=R_{p}+P_{p}\left(R_{i}, S_{i}\right), \\
Y_{p}=S_{p}+Q_{p}\left(R_{i}, S_{i}\right),
\end{gathered}
$$

where $\boldsymbol{\Phi}(\boldsymbol{u})=\left(P_{1} Q_{1} \cdots P_{N} Q_{N}\right)^{\mathrm{T}}$ and $P_{p}, Q_{p}$ are third-order polynomials. More explicitly, for the undamped case, the normal transform writes:

$$
\begin{aligned}
X_{p}= & R_{p}+\sum_{i=1}^{N} \sum_{j \geq i}^{N}\left(a_{i j}^{p} R_{i} R_{j}+b_{i j}^{p} S_{i} S_{j}\right) \\
& +\sum_{i=1}^{N} \sum_{j \geq i}^{N} \sum_{k \geq j}^{N} r_{i j k}^{p} R_{i} R_{j} R_{k} \\
& +\sum_{i=1}^{N} \sum_{j=1}^{N} \sum_{k \geq j}^{N} u_{i j k}^{p} R_{i} S_{j} S_{k}, \\
Y_{p}= & S_{p}+\sum_{i=1}^{N} \sum_{j=1}^{N} \gamma_{i j}^{p} R_{i} S_{j}+\sum_{i=1}^{N} \sum_{j \geq i}^{N} \sum_{k \geq j}^{N} \mu_{i j k}^{p} S_{i} S_{j} S_{k} \\
& +\sum_{i=1}^{N} \sum_{j=1}^{N} \sum_{k \geq j}^{N} v_{i j k}^{p} S_{i} R_{j} R_{k},
\end{aligned}
$$

where the coefficients $\left(a_{i j}^{p}, b_{i j}^{p}, \gamma_{i j}^{p}, r_{i j k}^{p}, u_{i j k}^{p}, \mu_{i j k}^{p}\right.$, $v_{i j k}^{p}$ ) have analytic expressions [25] that are tuned so as to cancel all nonresonant terms, as explained in Sect. 2.2. ${ }^{2}$ After application of the nonlinear coordinate change (31a), (31b), the dynamics are expressed with the normal variables $\left(R_{p}, S_{p}\right)$ and are substantially simplified. Moreover, it opens the doors to reduced-order modeling since in the normal dynamics, all invariant-breaking terms have been cancelled; see, e.g., [25-28]. In the case where no internal resonance exists between the eigenfrequencies

\footnotetext{
${ }^{2}$ The notations $R_{p}$ and $P_{p}$, kept from the formalism of [25, 26], must not be confused with the polynomials $\boldsymbol{R}$ and $\boldsymbol{P}$ of Sect. 2.2.
} 
$\left\{\omega_{p}\right\}_{p=1, \ldots, N}$ of the system (28), the normal dynamics can be reduced to a single master coordinate, say $\left(R_{p}, S_{p}\right)$, by simply cancelling all the others normal coordinates: $\forall k \neq p, R_{k}=S_{k}=0$. Substitution in (31a), (31b) allows to recover the geometry of the $p$ th NNM in phase space (up to order three):

$$
\begin{aligned}
\forall k \neq p: \quad X_{k}= & a_{p p}^{k} R_{p}^{2}+b_{p p}^{k} S_{p}^{2}+r_{p p p}^{k} R_{p}^{3} \\
& +u_{p p p}^{k} R_{p} S_{p}^{2}, \\
Y_{k}= & \gamma_{p p}^{k} R_{p} S_{p}+\mu_{p p p}^{k} S_{p}^{3}+v_{p p p}^{k} S_{p} R_{p}^{2},
\end{aligned}
$$

and the dynamics on the manifold is governed by:

$$
\ddot{R}_{p}+\omega_{p}^{2} R_{p}+\left(A_{p p p}^{p}+h_{p p p}^{p}\right) R_{p}^{3}+B_{p p p}^{p} R_{p} \dot{R}_{p}^{2}=0,
$$

where the introduced $A_{p p p}^{p}$ and $B_{p p p}^{p}$ terms are byproducts of the normal form transform; their general expressions read:

$$
\begin{aligned}
& A_{i j k}^{p}=\sum_{l \geq i}^{N} g_{i l}^{p} a_{j k}^{l}+\sum_{l \leq i} g_{l i}^{p} a_{j k}^{l}, \\
& B_{i j k}^{p}=\sum_{l \geq i}^{N} g_{i l}^{p} b_{j k}^{l}+\sum_{l \leq i} g_{l i}^{p} b_{j k}^{l} .
\end{aligned}
$$

All these results will now be applied to a two-dofs mechanical system. Invariant manifolds and normal dynamics up to order three as expressed in Eqs. (32a), (32b) and (33) will allow assessment of the accuracy of the third-order asymptotic expansion, respectively, on the geometry of the manifolds in phase space, and on the backbone curve of the reduced dynamics.

\subsection{Example study}

Let us consider the mechanical system sketched in Fig. 2 and composed of a mass connected to two nonlinear springs. The equations of motion reads:

$$
\begin{gathered}
\ddot{X}_{1}+\omega_{1}^{2} X_{1}+\frac{\omega_{1}^{2}}{2}\left(3 X_{1}^{2}+X_{2}^{2}\right)+\omega_{2}^{2} X_{1} X_{2} \\
+\frac{\omega_{1}^{2}+\omega_{2}^{2}}{2} X_{1}\left(X_{1}^{2}+X_{2}^{2}\right)=0
\end{gathered}
$$

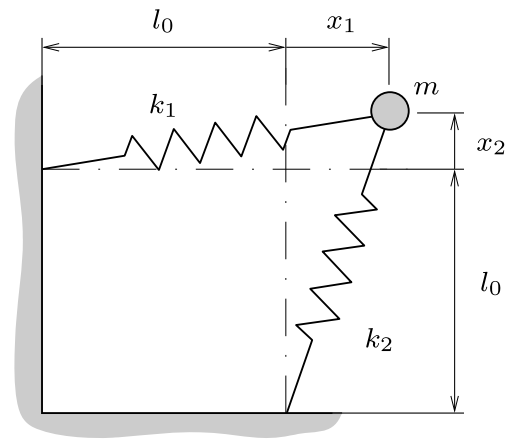

Fig. 2 Sketch of the two-dofs system considered. Mass $m$ is connected to two nonlinear springs

$$
\begin{gathered}
\ddot{X}_{2}+\omega_{2}^{2} X_{2}+\frac{\omega_{2}^{2}}{2}\left(3 X_{2}^{2}+X_{1}^{2}\right)+\omega_{1}^{2} X_{1} X_{2} \\
+\frac{\omega_{1}^{2}+\omega_{2}^{2}}{2} X_{2}\left(X_{1}^{2}+X_{2}^{2}\right)=0
\end{gathered}
$$

where $X_{1}=x_{1} / l_{0}, X_{2}=x_{2} / l_{0}$ are dimensionless displacements of the mass in the horizontal and vertical directions ( $l_{0}$ is the common free length of the springs). The nonlinear constitutive law of the springs is chosen analogous to a geometrically nonlinear Green-Lagrange strain-displacement law. It depends on stiffness constants $k_{1}$ and $k_{2}$ and is defined in [25]. The two eigenfrequencies $\omega_{1}=\sqrt{k_{1} / m}$ and $\omega_{2}=\sqrt{k_{2} / m}$ fully parameterize the nonlinear problem.

Regions of hardening/softening behavior of this system, as functions of $\left(\omega_{1}, \omega_{2}\right)$, have already been studied in [25]. Here, three cases are selected for computations:

- Case 1 , for $\left(\omega_{1}, \omega_{2}\right)=(\sqrt{1.7}, \sqrt{6})$.

- Case 2, for $\left(\omega_{1}, \omega_{2}\right)=(\sqrt{3}, 1)$.

- Case 3, for $\left(\omega_{1}, \omega_{2}\right)=(\sqrt{0.5}, \sqrt{6})$.

A global picture of the dynamics for case 1 is shown in Fig. 3. Frequency-amplitude relationships (so-called backbone curves) are reported for modes 1 and 2. A reference solution is found by numerical continuation of periodic orbits from the full system (35a), (35b), and realized with the software MANLAB [29]. MANLAB uses an asymptotic numerical method for continuation of amplitudes of the Fourier coefficients of the solution derived from a harmonic balance method [30] and the stability of the periodic orbits is computed with a modified Hill algo- 
(a)

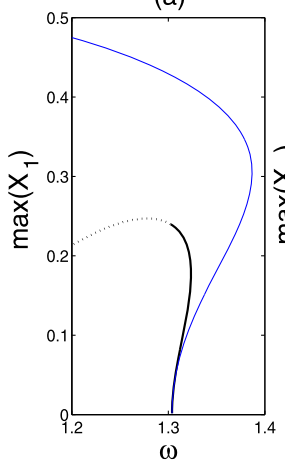

(b)

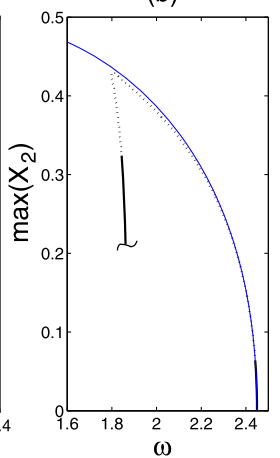

(c)

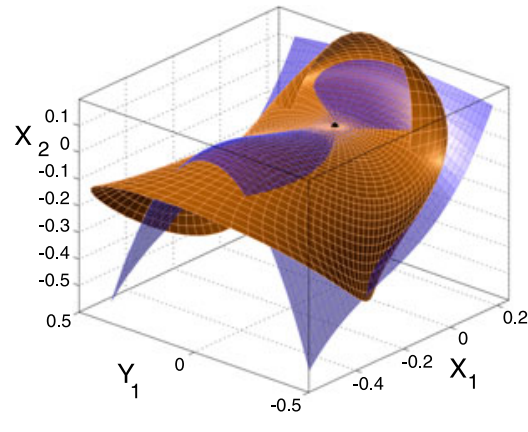

(d)

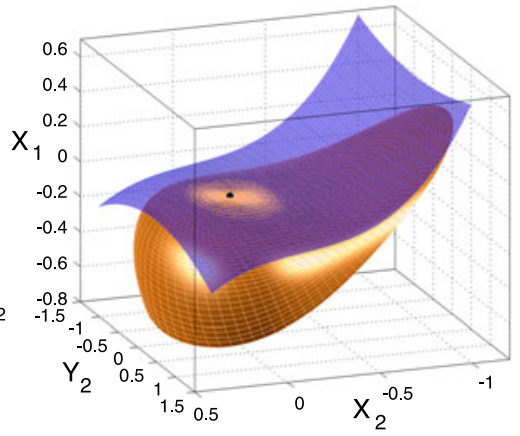

Fig. 3 Backbone curves and NNMs for the two-dofs system considered, case 1: $\left(\omega_{1}, \omega_{2}\right)=(\sqrt{1.7}, \sqrt{6}) .(\mathbf{a})-(\mathbf{b})$ backbone curves for modes 1 and 2. Comparison between the exact solution, computed by numerical continuation on the original system (black, thick line) and the third-order approximation provided

rithm [31]. The reference solution is compared to the backbone curve obtained numerically by continuation of periodic orbits on the reduced system, expressed by Eq. (33). Amplitude-frequency relationships are given for the maximum amplitude of the periodic orbits, i.e., the maximum value of $X_{1}$ for mode 1 , and of $X_{2}$ for mode 2 . The geometry of the two NNMs is also shown. Once again, the exact solution (periodic orbits) is compared to the third-order approximation given by Eqs. (32a), (32b). The geometry of the exact manifolds shows that they fold for a given amplitude: 0.247 for mode 1 , and 0.43 for mode 2. This folding corresponds exactly to the point on the backbone curve where an horizontal tangency is found for mode 1 , and for mode 2 the point where the amplitude suddenly decreases. For mode 2 , one can observe on the amplitude-frequency relationship that until that folding point, the thirdorder approximation gives a quantitatively excellent approximation. However, mode 2 loses stability very quickly for an amplitude: $\max \left(X_{2}\right)=0.06$, whereas the asymptotic solution predicts stable solutions. For mode 1, one can observe that from an amplitude of 0.1 , the third-order approximation departs from the exact solution. Then it misses the folding point and is thus completely unreliable for amplitudes over 0.25 .

Cases 2 and 3 of the same system are inspected in Fig. 4. Case 2 shows a phenomenology comparable to case 1 , by inverting the roles of mode 1 and mode 2 . The shapes of the invariant manifolds are by Normal Form (blue, thin line). Unstable solutions are represented by dots. (c) First NNM in space $\left(X_{1}, Y_{1}, X_{2}\right)$. Comparison of exact solution (periodic orbits, $r e d$ ) and third-order approximation (light blue). (d) idem for the second NNM in space $\left(X_{2}, Y_{2}, X_{1}\right)$

very similar: Fig. 4(c) representing the second NNM of case 2 resembles mode 1 of case 1 , and the same is true for the subsequent mode. The backbone curves also show comparable behavior, with a sharp folding for mode 1 and a vertical tangency for mode 2 . The real normal form approximation gives an excellent result for mode 1 except for the stability that is not correctly predicted, whereas for mode 2 , the third-order approximation departs from the exact solution from an amplitude of 0.2 , and then misses completely the folding point for $\max \left(X_{2}\right)=0.26$. Case 3 shows a different behavior. In particular, the backbone curve for mode 1 shows a variation in its softening behavior that is enhanced for small amplitudes. Then a folding of the manifold at $\max \left(X_{1}\right)=0.4$ is observed. The geometry of the first NNM is represented in Fig. 4(f), showing that the third-order approximation fails to catch its complex shape, resulting in an approximated backbone curve that departs from the exact solution at the point where the softening behavior decreases, i.e., for $\max \left(X_{1}\right)=0.27$. On the other hand, the approximation for mode 2 is reliable until $\max \left(X_{2}\right)=0.3$.

\subsection{Validity limits}

The criterion proposed in Sect. 2 is now applied to the two-dofs system. The matrix $\boldsymbol{V}=\boldsymbol{I}+\partial \boldsymbol{\Phi}(\boldsymbol{u})$ depends on the four normal variables $\left(R_{1}, S_{1}, R_{2}, S_{2}\right)$. Following the notations introduced in Sect. 4.1, we can write explicitly: 
(a)
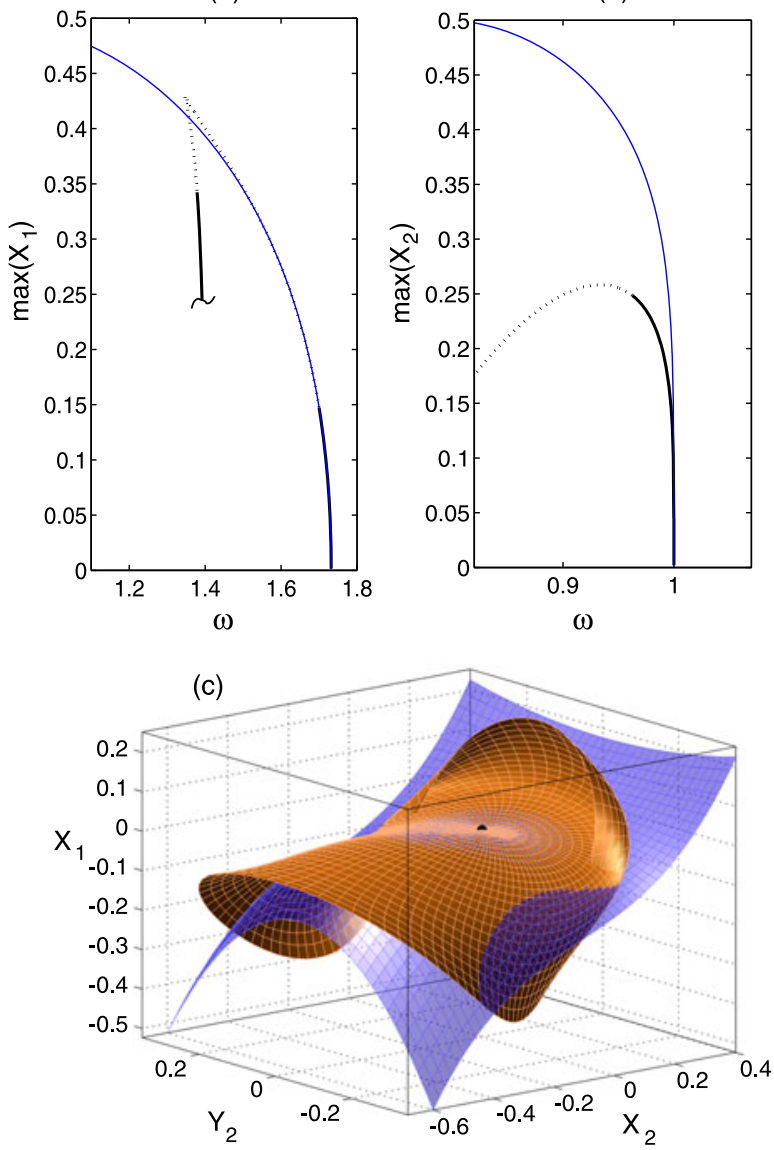

Fig. 4 Backbone curves and NNMs for the two-dofs system considered, $(\mathbf{a}-\mathbf{c})$ case $2,\left(\omega_{1}, \omega_{2}\right)=(\sqrt{3}, 1),(\mathbf{d}-\mathbf{f})$ case 3 , $\left(\omega_{1}, \omega_{2}\right)=(\sqrt{0.5}, \sqrt{6})$. (a, b) backbone curves for mode 1 and 2, case 2. Comparison between the exact solution, computed by numerical continuation on the original system (black, (d)

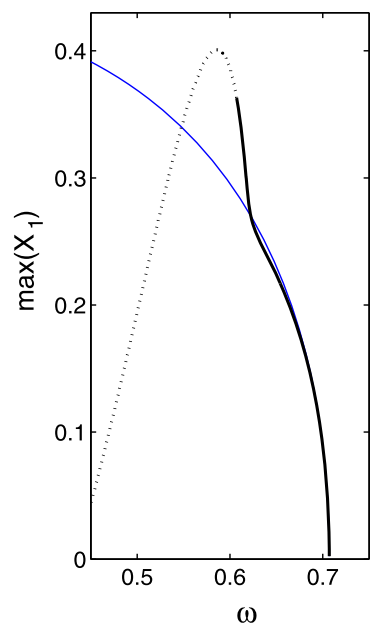

(e)
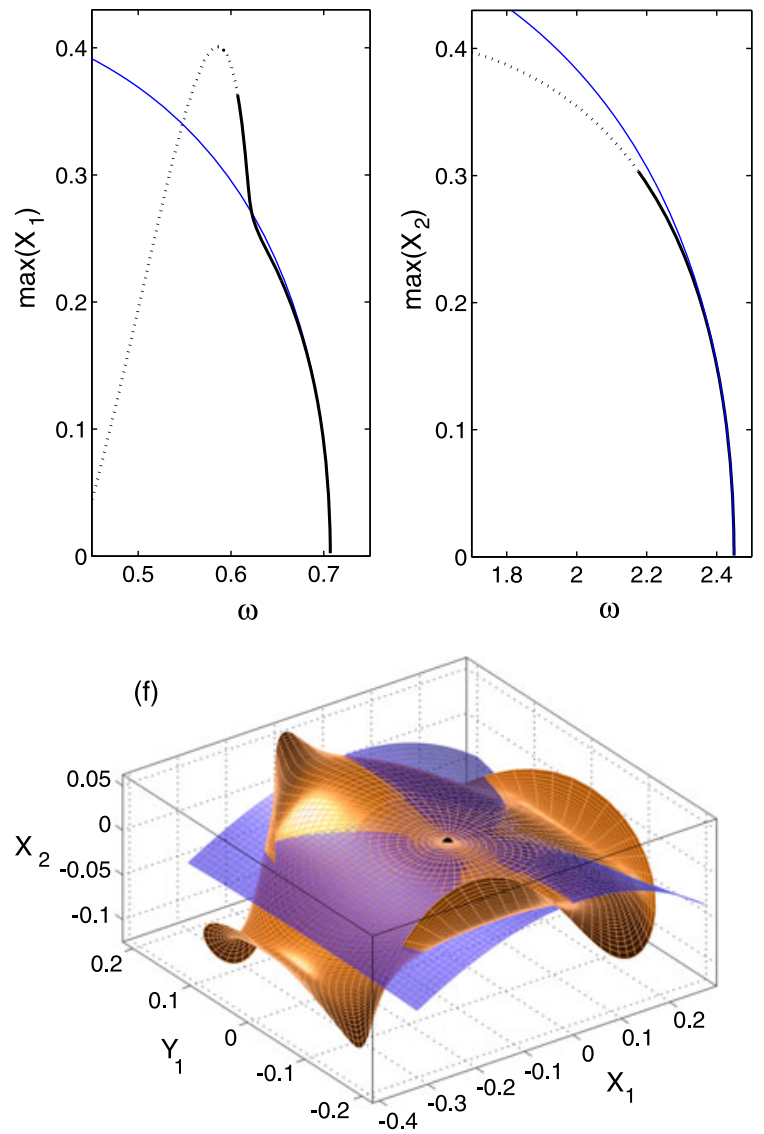

thick line) and the third-order approximation provided by Normal Form (blue, thin line). Unstable solutions are represented by dots. (c) second NNM plotted in $\left(X_{2}, Y_{2}, X_{1}\right)$. (d-e) backbone curves for modes 1 and 2, case 3. (f) geometry of the first NNM, case 3

$\boldsymbol{V}\left(R_{1}, S_{1}, R_{2}, S_{2}\right)=\left[\begin{array}{cccc}1+\partial P_{1} / \partial R_{1} & \partial P_{1} / \partial S_{1} & \partial P_{1} / \partial R_{2} & \partial P_{1} / \partial S_{2} \\ \partial Q_{1} / \partial R_{1} & 1+\partial Q_{1} / \partial S_{1} & \partial Q_{1} / \partial R_{2} & \partial Q_{1} / \partial S_{2} \\ \partial P_{2} / \partial R_{1} & \partial P_{2} / \partial S_{1} & 1+\partial P_{2} / \partial R_{2} & \partial P_{2} / \partial S_{2} \\ \partial Q_{2} / \partial R_{1} & \partial Q_{2} / \partial S_{1} & \partial Q_{2} / \partial R_{2} & 1+\partial Q_{2} / \partial S_{2}\end{array}\right]$

The determinant of $\boldsymbol{V}$ is computed on a four-dimensional grid, which can give rise to important computational times for fine grids. In order to represent the solution, two coordinates $\rho_{1}$ and $\rho_{2}$ are introduced as:

$\rho_{1}=\sqrt{R_{1}^{2}+\frac{S_{1}^{2}}{\omega_{1}^{2}}}$,

$$
\rho_{2}=\sqrt{R_{2}^{2}+\frac{S_{2}^{2}}{\omega_{2}^{2}}} .
$$

They can be viewed as amplitudes of the periodic orbits and are easily related to the amplitudes of motions used for parameterizing the backbone curves. Moreover, they allow representation of $\Delta=\operatorname{det}(\boldsymbol{V})$ in a 
(a)

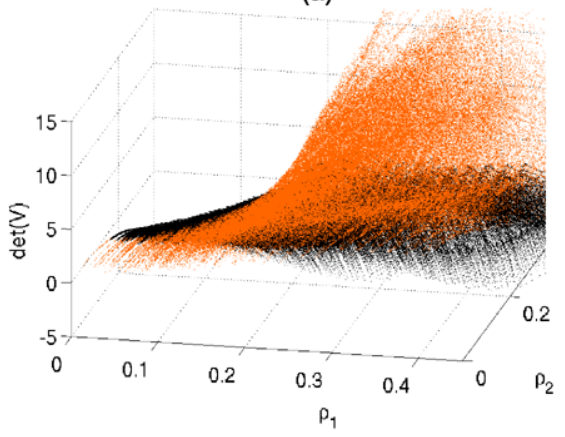

(b)

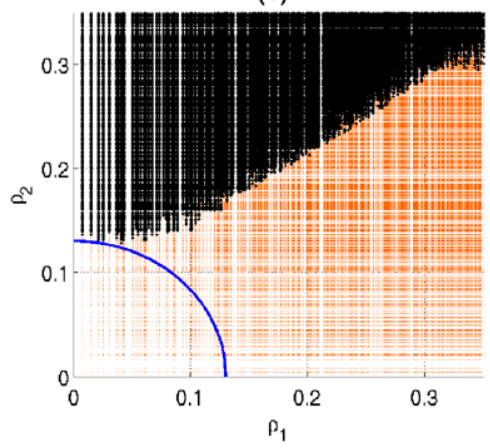

(c)

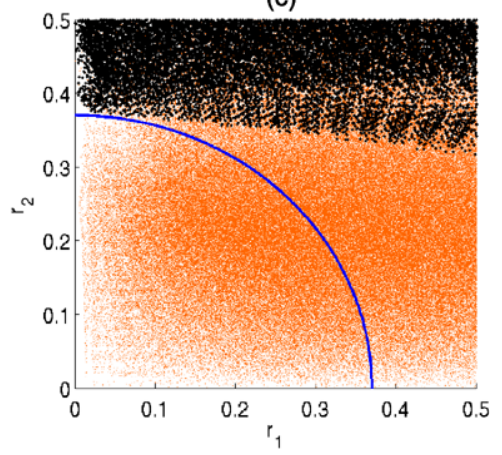

Fig. 5 Validity limit criterion for the two-dofs example, case 1. (a) Three-dimensional view of $\operatorname{det}(\boldsymbol{V})$ as function of $\left(\rho_{1}, \rho_{2}\right)$. Positive values of $\operatorname{det}(\boldsymbol{V})$ are plotted with orange points while negative values with black points. (b) Upper view of $\operatorname{det}(\boldsymbol{V})$ as

two-dimensional space. Finally, the nonlinear change of coordinates (31a), (31b) is applied to each point of the grid to recover the original displacements and velocities. The value of $\Delta$ is also represented on a twodimensional grid by using the reduced coordinates:

$$
\begin{aligned}
& r_{1}=\sqrt{X_{1}^{2}+\frac{Y_{1}^{2}}{\omega_{1}^{2}}}, \\
& r_{2}=\sqrt{X_{2}^{2}+\frac{Y_{2}^{2}}{\omega_{2}^{2}}} .
\end{aligned}
$$

Figure 5 shows the obtained results for case 1. First, a three-dimensional view of the values of $\operatorname{det}(\boldsymbol{V})$ is represented in Fig. 5(a). One can observe the very complex shape of the surface in the plane $\left(\rho_{1}, \rho_{2}\right)$, which is so irregular that it tends to occupy a volume rather than a surface, making representation quite difficult. The following features are worth mentioning: when $\rho_{1}$ and $\rho_{2}$ tend to zero, $\operatorname{det}(\boldsymbol{V})$ tends to 1 , in the line of its definition. For $\rho_{2}=0$ and increasing values of $\rho_{1}$, one can observe that $\operatorname{det}(\boldsymbol{V})$ tends to larger and larger positive values. On the other hand, for $\rho_{1}=0$ and increasing values of $\rho_{2}$, the values of $\operatorname{det}(\boldsymbol{V})$ decreases and then becomes negative. In the remainder of the plane $\left(\rho_{1}, \rho_{2}\right), \operatorname{det}(\boldsymbol{V})$ takes very irregular values so that numerous cancellation points are obtained.

A top view of the plot is shown in Fig. 5(b) so as to better visualize the cancellation points of $\operatorname{det}(\boldsymbol{V})$. The upper bound for validity limits of the third-order real normal form is defined by the largest ball for which $\operatorname{det}(\boldsymbol{V})$ does not cancel. Defining the vector $\boldsymbol{\rho}$ as an function of $\left(\rho_{1}, \rho_{2}\right)$, positive points in orange, negative in black. A circle of radius $\rho=0.13$ is superimposed. (c) $\operatorname{det}(\boldsymbol{V})$ as function of $\left(r_{1}, r_{2}\right)$, in order to recover original coordinates. A circle of radius $r=0.37$ is superimposed

element of the plane $\left(\rho_{1}, \rho_{2}\right)$, one can see on Fig. 5(b) that the largest subset without cancellation is given by the circle of radius $\rho=R_{\lim }=0.13$. Finally, Fig. 5(c) shows the values of $\operatorname{det}(\boldsymbol{V})$ after the nonlinear change of coordinates has been applied, so as to get a better quantitative comparison with the global dynamics exhibited in the previous section. The nonlinear change of coordinates severely distorts the initial regular grid defined for $\left(R_{1}, S_{1}, R_{2}, S_{2}\right)$. In the original dynamical variables $\left(X_{1}, Y_{1}, X_{2}, Y_{2}\right)$, the largest subset for which $\operatorname{det}(\boldsymbol{V})$ does not cancel is given by the ball of radius $r=0.37$. Compared with the quantitative description of the dynamics and its third-order approximation given in Sect. 4.2, one can see that the upper bound given by the proposed criterion gives a coherent value. As observed in that case, the asymptotic solution for mode 1 departs form the exact solution for an amplitude of 0.1 , while for mode 2 a good estimate was found until 0.43 , but the stability was not correctly predicted. Hence, limiting the validity of the normal form solution to an amplitude of 0.37 is fully coherent with that analysis.

The criterion for validity limits is tested on cases 2 and 3 in Fig. 6. For case 2, the upper bound is found to be for an amplitude (in original coordinates) of 0.26 . This result is completely in the line of the dynamical solutions inspected in Sect. 4.2, where mode 2 was shown to fold for that amplitude, so that the asymptotic solution is completely unreliable for larger amplitudes. Finally, for case 3, the validity limit given by the criterion is a ball of the radius $r=0.37$, which appears to be an upper bound as the asymptotic backbone 
Fig. 6 Validity limit criterion for the two-dofs example, (a) case 2,

(b) case 3. Upper view of $\operatorname{det}(\boldsymbol{V})$ as function of $\left(r_{1}, r_{2}\right)$, positive points in orange, negative in black. A circle of radius $r=0.26$ is superimposed for case 2 in (a); $r=0.37$ for case 3 in (b) (a)

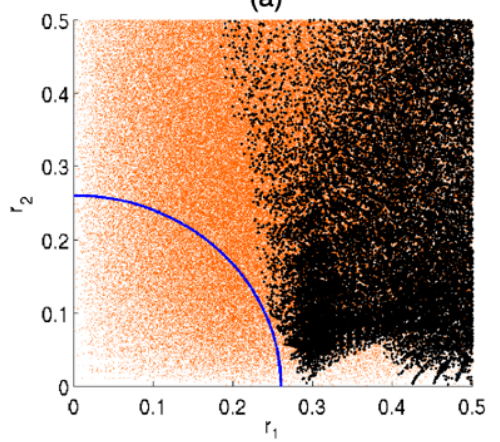

(b)

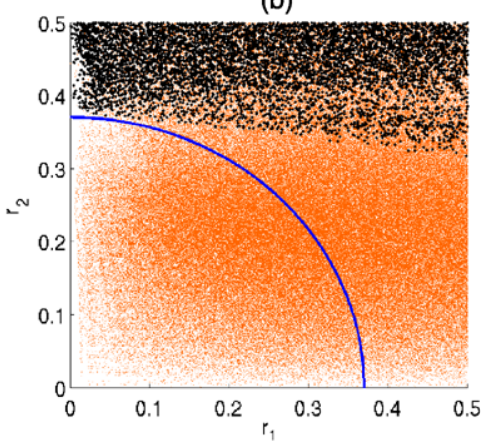

curves for mode 1 and mode 2 depart from the correct solution for amplitudes around 0.3. Once again, the criterion gives a very coherent value for obtaining a validity limit for normal form transformations.

\subsection{Effect of damping}

The effect of adding a linear viscous damping term to the equations of motion on the validity limit is now studied. For that purpose, Eqs. (35a), (35b) are modified by adding a term of the form $2 \xi_{i} \omega_{i} \dot{X}_{i}, i=1,2$, on each equation. The nonlinear change of coordinates, Eqs. (31a), (31b), is modified following a procedure that is fully explained in [26]. The presence of the damping slightly modifies the coefficients of the normal form, and may also have an influence on the type of nonlinearity; see [26] for a full discussion including examples.

The criterion for the upper bound is computed as in Sect. 4.3, with the modified coefficients now depending on damping. Results are shown in Fig. 7, for three increasing values of the damping, where we have selected the same damping ratio for the two oscillators: for $\mathrm{i}=1,2, \xi_{i}=10^{-3}, \xi_{i}=10^{-2}$, and $\xi_{i}=10^{-1}$. One can observe that:

- for case 1 , the validity limit increases. For $\xi_{i}=10^{-3}$ and $\xi_{i}=10^{-2}$, we have $\rho_{\mathrm{lim}}=0.135$, and for $\xi_{i}=$ $10^{-1}, \rho_{\text {lim }}=0.21$.

- for case 2 , the validity limit very slightly increases from $\rho_{\text {lim }}=0.34$ to $\rho_{\text {lim }}=0.37$.

- for case 2 , the validity limit decreases from 0.41 to 0.38 .

As already mentioned in [26], the effect of the damping on the normal form coefficients is slight as long as small damping ratios are taken into account.
However, one must keep in mind that these slight modifications have a quantitative effect on NNM-based reduced-order models for the forced response of continuous structures or on the type of nonlinearity. In the case of predicting a limit value for those analytical asymptotic developments, the main conclusion is that only slight changes are observed by taking dissipation into account, so that the order of magnitude given by the conservative case is maintained. The fact that the upper bound increases or decreases with the damping seems to be problem-dependent and does not lend itself to a simple physical interpretation.

\subsection{Normal forms and nonlinear normal modes}

To conclude this analysis, a more thorough comparison of real normal form with NNMs computation using the center manifold technique [11-13], as proposed by Shaw and Pierre [32, 33], is here given. For the sake of clarity, the original dynamical equations (1) are restricted to a two dofs problem, so that $X=$ $\left(\begin{array}{llll}X_{1} & Y_{1} & X_{2} & Y_{2}\end{array}\right)^{\mathrm{T}}=\left(\begin{array}{ll}\boldsymbol{X}_{1} & \boldsymbol{X}_{2}\end{array}\right)^{\mathrm{T}}$, where $\boldsymbol{X}_{1}=\left(\begin{array}{lll}X_{1} & Y_{1}\end{array}\right)^{\mathrm{T}}$ (resp. $\left.\boldsymbol{X}_{2}=\left(X_{2} Y_{2}\right)^{\mathrm{T}}\right)$ is the vector of coordinatesdisplacement and velocity-related to mode 1 (resp. mode 2). Nonlinear normal modes (NNMs) are exhibited using the technique of the center manifold theorem by separating the problem into:

$\dot{X}_{1}=A_{1} X_{1}+g_{1}\left(X_{1}, X_{2}\right)$,

$\dot{X}_{2}=A_{2} X_{2}+g_{2}\left(X_{1}, X_{2}\right)$.

For computing the first NNM, one wants to eliminate properly the second coordinate from (41a) without simply cancelling $\boldsymbol{X}_{2}$. This is realized by postulating a functional relationship:

$X_{2}=\psi_{1}\left(X_{1}\right)$ 

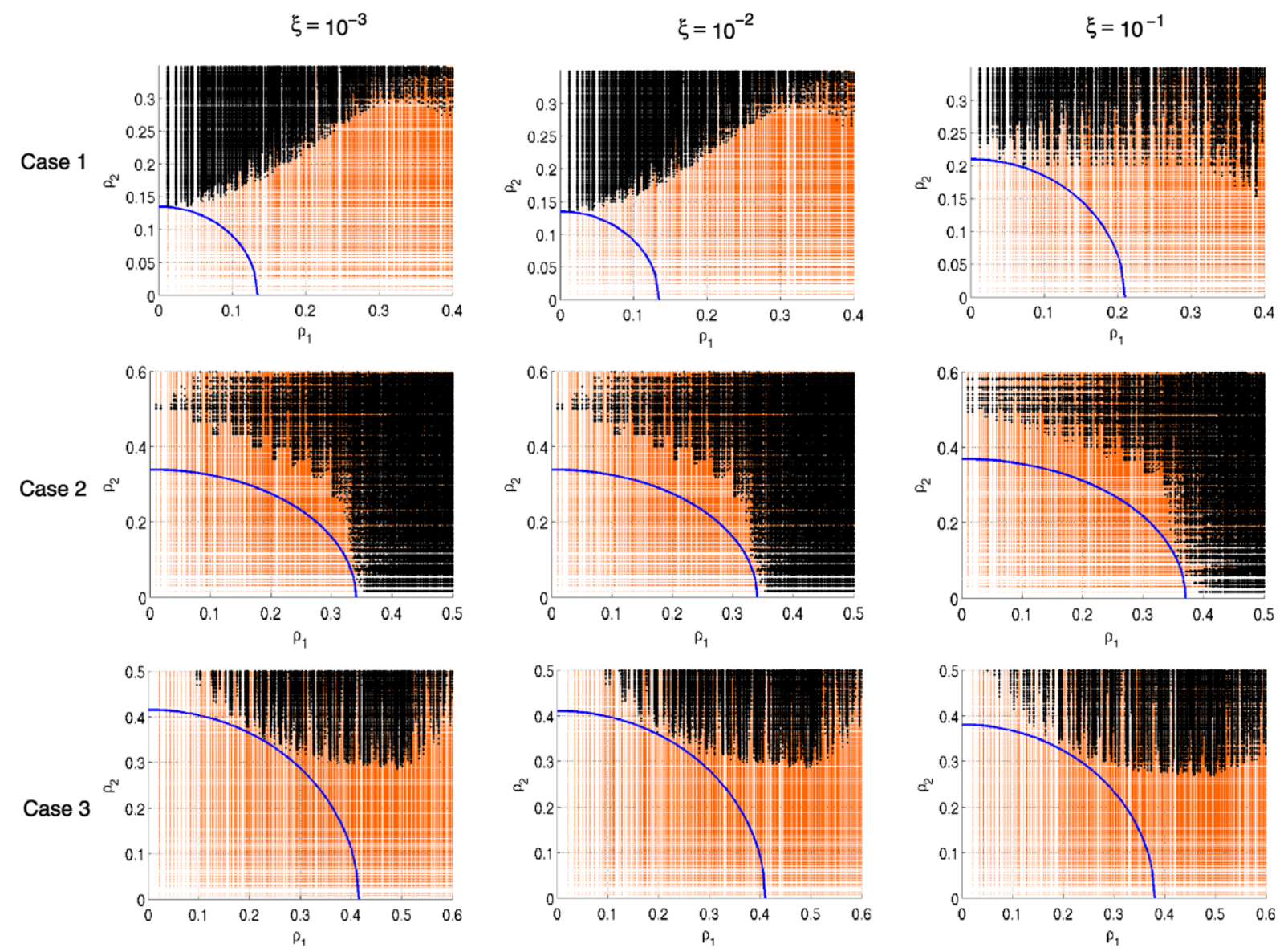

Fig. 7 Effect of the damping on the criterion for validity limit. Upper view of $\operatorname{det}(\boldsymbol{V})$ as a function of $\left(\rho_{1}, \rho_{2}\right)$, positive points in orange, negative in black. A circle is superimposed to mark the ball of maximum radius without cancellation of

Taking the derivative (42) with respect to time, and eliminating time using the relationships provided by (41a), (41b) allows exhibiting the equation governing the geometry of the first invariant manifold (the first $\mathrm{NNM}$ ) in phase space. Solving this equation gives the unknown $\psi_{1}$, and thus the first NNM.

The relationship between NNMs and normal form theory can be formally exhibited as follows. Equation (2) can be split into two parts as:

$$
\begin{aligned}
& \boldsymbol{X}_{1}=\boldsymbol{U}_{1}+\boldsymbol{\Phi}_{1}\left(\boldsymbol{U}_{1}, \boldsymbol{U}_{2}\right), \\
& \boldsymbol{X}_{2}=\boldsymbol{U}_{2}+\boldsymbol{\Phi}_{2}\left(\boldsymbol{U}_{1}, \boldsymbol{U}_{2}\right) .
\end{aligned}
$$

In this formalism, restraining motions to the first NNM is simply obtained by cancelling the normal variable related to the second NNM: $\boldsymbol{U}_{2}=0$ [25]. Hence, the
$\operatorname{det}(\boldsymbol{V})$. Columns: increasing values of the damping: $\xi=10^{-3}$, $10^{-2}$ and $10^{-1}$. Raws: case $1:\left(\omega_{1}, \omega_{2}\right)=(\sqrt{1.7}, \sqrt{6})$, case 2 : $\left(\omega_{1}, \omega_{2}\right)=(\sqrt{3}, 1)$, and case $3:\left(\omega_{1}, \omega_{2}\right)=(\sqrt{0.5}, \sqrt{6})$

equivalent formulation of the functional relationship used to define the first NNM; Eq. (42) is given by:

$$
\begin{aligned}
& \boldsymbol{X}_{1}=\boldsymbol{U}_{1}+\boldsymbol{\Phi}_{1}\left(\boldsymbol{U}_{1}, 0\right), \\
& \boldsymbol{X}_{2}=\boldsymbol{\Phi}_{2}\left(\boldsymbol{U}_{1}, 0\right)
\end{aligned}
$$

One can observe that for recovering $\boldsymbol{\psi}_{1}$, local inversion of (44a) has to be computed before inserting in (44b).

Now, one wants to recover, if possible, the upper bound exhibited in the previous sections on normal form when performing NNM computations, as one would be interested, e.g., when performing numerical simulations and asymptotic reduced-order models (ROMs), in the validity limit of its asymptotic expansion used to compute a NNM-based ROM. Taking the 
derivative of (42) with respect to time yields

$\dot{\boldsymbol{X}}_{2}=\frac{\partial \boldsymbol{\psi}_{1}}{\partial \boldsymbol{X}_{1}} \dot{\boldsymbol{X}}_{1}$

Taking also the derivative of Eqs. (43a), (43b) with respect to time allows eliminating $\dot{\boldsymbol{X}}_{1}$ and $\dot{\boldsymbol{X}}_{2}$ from Eq. (45), leading to

$$
\begin{aligned}
\dot{\boldsymbol{U}}_{2} & +\frac{\partial \boldsymbol{\Phi}_{2}}{\partial \boldsymbol{U}_{1}} \dot{\boldsymbol{U}}_{1}+\frac{\partial \boldsymbol{\Phi}_{2}}{\partial \boldsymbol{U}_{2}} \dot{\boldsymbol{U}}_{2} \\
& =\frac{\partial \boldsymbol{\psi}_{1}}{\partial \boldsymbol{X}_{1}}\left[\dot{\boldsymbol{U}}_{1}+\frac{\partial \boldsymbol{\Phi}_{1}}{\partial \boldsymbol{U}_{1}} \dot{\boldsymbol{U}}_{1}+\frac{\partial \boldsymbol{\Phi}_{1}}{\partial \boldsymbol{U}_{2}} \dot{\boldsymbol{U}}_{2}\right] .
\end{aligned}
$$

Now restriction to the first NNM is obtained by cancelling normal variables of the second NNM: $\boldsymbol{U}_{2}=0$ and $\dot{\boldsymbol{U}}_{2}=0$. Eliminating $\dot{\boldsymbol{U}}_{1}$, the previous equation reduces to

$$
\frac{\partial \boldsymbol{\Phi}_{2}}{\partial \boldsymbol{U}_{1}}=\frac{\partial \boldsymbol{\psi}_{1}}{\partial \boldsymbol{X}_{1}}\left[\boldsymbol{I}_{2}+\frac{\partial \boldsymbol{\Phi}_{1}}{\partial \boldsymbol{U}_{1}}\right],
$$

where $I_{2}$ is the $2 \times 2$ identity matrix. Hence, for obtaining an expression of $\boldsymbol{\psi}_{1}(\mathrm{NNM})$ as function of $\boldsymbol{\Phi}_{1}$ and $\boldsymbol{\Phi}_{2}$ (normal form expression), one has to invert the previous relationship, which is possible if and only if $\boldsymbol{I}_{2}+\frac{\partial \boldsymbol{\Phi}_{1}}{\partial \boldsymbol{U}_{1}}$ does not show any cancellation. This expression is exactly the same as our criterion used for giving an upper bound, except that it is restricted to a two-dimensional problem whereas the original criteria was fully four-dimensional with all cross-derivatives involved. The expression $\boldsymbol{I}_{2}+\frac{\partial \boldsymbol{\Phi}_{1}}{\partial \boldsymbol{U}_{1}}$ corresponds to the $2 \times 2$ upper left block of $\boldsymbol{V}$ expressed in Eq. (36). The same reasoning using the second NNM would have led us to consider $\boldsymbol{I}_{2}+\frac{\partial \boldsymbol{\Phi}_{2}}{\partial \boldsymbol{U}_{2}}$ that corresponds to the $2 \times 2$ lower right block of $\boldsymbol{V}$. Hence, using the NNMs only for testing the upper bound will lead to erroneous result, as a very restrictive criterion will be computed instead of the global one. This calculation points out the fact that the nonlinear change of coordinates for reducing the problem has to be computed on a ball of the complete phase space around the origin, hence involving all variables. Projecting first the dynamical equations onto two-dimensional invariant manifolds has to be done with care when one wants to recover global quantities involving the complete phase space. The restriction underlined here is somehow equivalent to the restriction one has to face when building out a complete change of coordinates, from the initial modal ones, to the coordinates describing the invariant manifolds. Shaw and Pierre proposed in [33] to gather all functional relationships of the form (42) in order to define this change of coordinates. Unfortunately, this idea relies on linear concepts, and thus gives an incorrect formulation, as underlined in [25, 34]. The correct formulation is given by the normal form which explicitly computes all nonlinear terms, as shown in [25]. The same process is here underlined, as using a simplified criterion for the upper bound for the validity limit of asymptotic expansion leads to use a restrictive criterion conducting to erroneous results.

A less restrictive point of view is to consider the full $4 \times 4$ criterion expressed by $\boldsymbol{V}$ in (36), and to set $R_{2}=S_{2}=0$ (resp. $R_{1}=S_{1}=0$ ) for considering the first (resp. the second) NNM. By doing so, $\boldsymbol{V}$ depends only on two variables, which renders the computations much more efficient. In particular, this method could be an alternative if one wants to get an upper bound for a $N$-dofs problem with $N$ large as, e.g., in [28] where ROMs are derived for shell vibrations problems with routinely $N=20$. In that case, computing the full criterion will lead to define a 40-dimensional grid, which will be impossible in a reasonable computation time. Hence, a simplified criterion, consisting in restraining to each NNM, and thus relying on 20 two-dimensional problems to compute, could be used to get an upper bound to assess the validity limit.

Let us denote $V_{1}=\operatorname{det}\left[V\left(R_{1}, S_{1}, 0,0\right)\right]$ and $V_{2}=$ $\operatorname{det}\left[\boldsymbol{V}\left(0,0, R_{2}, S_{2}\right)\right]$. They are represented as contour plots in Fig. 8 for case $1,\left(\omega_{1}, \omega_{2}\right)=(\sqrt{1.7}, \sqrt{6})$. $V_{1}$ shows positive values in the vicinity of the origin and a cancellation line crossing the $R_{1}$-axis for $R_{1}=-0.57$. If used as a criterion for the validity limit, we would have obtained a largely overstimated value, as the complete criterion shown in Fig. 5(b) predicts a ball of radius $\rho=0.13$. On the other hand, $V_{2}$ shows a zero-crossing value for small amplitudes, highlighted in Fig. 8(b). Hence, the upper bound can be assessed and is found numerically for $\rho_{1}=0.13$, which is exactly the value found by the 4-dimensional criterium. This is logical since the reduced criterion can be inferred from the results presented in Fig. 5(b) by selecting only the line $\rho_{2}=0$ (resp. $\rho_{1}=0$ ) for $V_{1}$ (resp. $V_{2}$ ). And for case 1 , the first cancellation point exhibited by Fig. 5 was found near the line $\rho_{1}=0$.

The same result is found for case 2 , where the cancellation point is found near the axis $\rho_{2}=0$, as it can be seen on Fig. 7 (note that the figure for the conservative case and the damped one with a damping ratio of $10^{-3}$ are coincident, so that the results for the conservative case can be inferred from those with small 

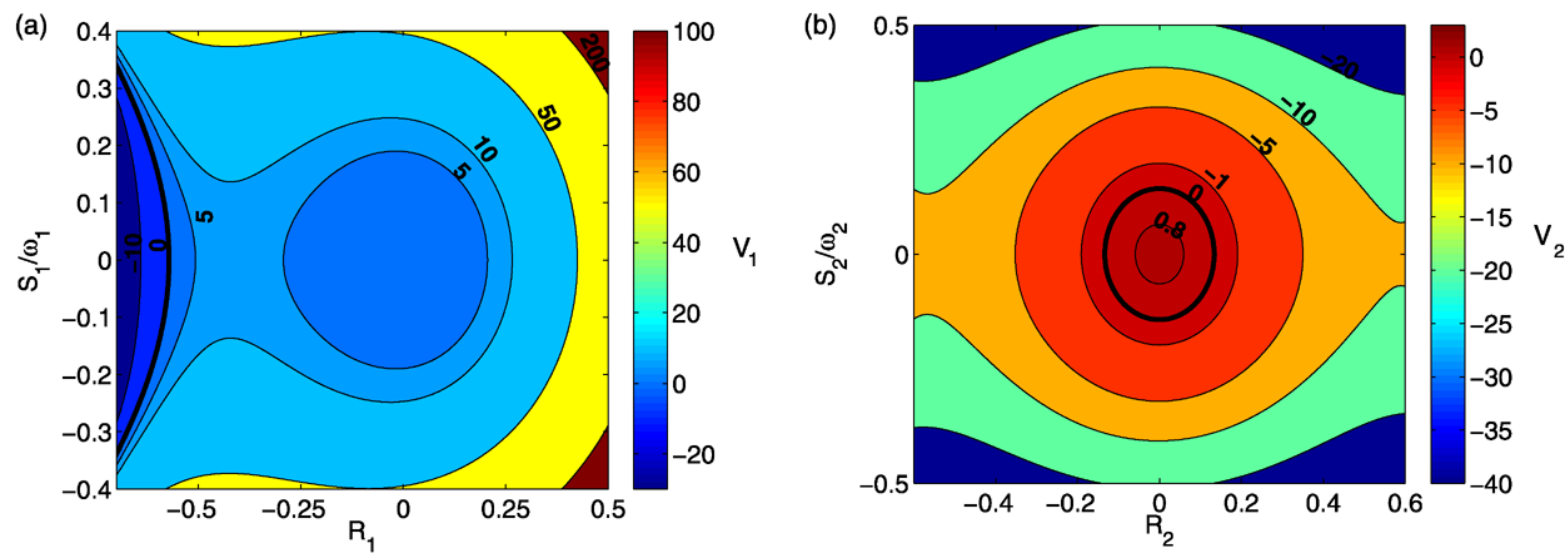

Fig. 8 Contour plots of (a) $V_{1}=\operatorname{det} \boldsymbol{V}\left(R_{1}, S_{1}, 0,0\right)$, and (b) $V_{2}=\operatorname{det} \boldsymbol{V}\left(0,0, R_{2}, S_{2}\right)$, for case 1

damping). Hence, computing $V_{2}$ for case 2 does not predict a correct validity limit. On the other hand, $V_{1}$ gives a validity limit of $\rho_{2}=0.34$, exactly the value given by the complete criterium.

Finally, case 3 shows a cancellation point being neither on $\rho_{2}=0$ axis, nor on $\rho_{1}=0$ (see Fig. 7). Hence, using the reduced criteria with $V_{1}$ and $V_{2}$ in case 3 leads to overestimate the upper bound. Numerical results show that the upper bound is found for $V_{1}$ with a value $\rho_{1}=0.68$, whereas the value given by $V_{2}$ is much larger. That has to be compared to the radius given by the full criterion: $\rho=0.42$. To conclude, these reduced criteria can be utilized in order to speed up the computations, as two-dimensional curves have to be computed instead of a $2 \mathrm{~N}$ dimensional problem in the general case. But one has to keep in mind that generally an overestimate will be given.

\section{Conclusion}

A criterion for assessing the validity limit of analytical approximate solutions obtained by means of a normal form transformation has been presented. It relies on the interpretation of the homological equations as an inversion procedure, hence allowing derivation of a nonvanishing quantity that has to be checked for the amplitudes of the normal coordinates. The criterion has to be checked order by order, in the line of normal form transforms that are successively computed by increasing orders of nonlinearity. The criterion has been tested on the Duffing equation together with a complex formulation of the normal form. For that case, computations of normal forms up to order 27 have been realized, showing that the validity criterion converges to the value of 1 , which is meaningful for a perturbative asymptotic solution where it is generally required that nonlinear terms sort according to increasing orders, which is realized as long as the variables are less than 1 . As the radius of the validity limit given by the criterion converge in a decreasing manner, it can be used for first orders, but gives in that case an upper bound for the validity limit. The criterion has then been tested on a two dofs system with real normal form transformations up to order 3. Dynamical solutions have been computed numerically for assessing the range of validity of the third-order normal form transform on the backbone curves of the system. Comparison with the upper bound provided by the criterion shows very good agreement. However, computation times can become rapidly prohibitive as they scale with the dimension of the phase space. Hence, reduced criterion and comparison with reduced-order models based on NNMs computations have been proposed. Finally, the case of adding linear viscous damping terms in the mechanical system has been considered, showing that its influence on the criterion for the validity limit is reduced to cases where damping is very large.

\section{Appendix A: Normal form of free Duffing equation up to degree 11}

We give the normal form for the Duffing equation up to degree 11 for illustrating the complexity of the calculations operated in Sect. 3.1. Note that calculations 
up to order 27 have been realized with the help of a symbolic calculator, however, results are not provided as they would cover the pages. The normal form of Eq. (16), up to degree 11, reads

$$
\begin{aligned}
\dot{u}= & -i \omega_{1} u_{1}+\frac{-\frac{147}{16} i c^{3} u_{1}{ }^{4} u_{2}{ }^{3}}{\omega_{1}{ }^{5}}+\frac{\frac{31035}{1024} i c^{4} u_{1}{ }^{5} u_{2}{ }^{4}}{\omega_{1}{ }^{7}} \\
& -\frac{\frac{887193}{8192} i c^{5} u_{1}{ }^{6} u_{2}{ }^{5}}{\omega_{1}{ }^{9}}+\frac{\frac{51}{16} i c^{2} u_{1}{ }^{3} u_{2}{ }^{2}}{\omega_{1}{ }^{3}} \\
& -\frac{3 / 2 i c u_{1}{ }^{2} u_{2}}{\omega_{1}}
\end{aligned}
$$

the second equation being the complex conjugate of this one. The corresponding normal transform $\boldsymbol{\Phi}_{1}(\boldsymbol{u})$ is given by

$$
\begin{aligned}
& \Phi_{1}(u)=-\frac{887193}{16384} \frac{c^{5} u_{1}{ }^{5} u_{2}{ }^{6}}{\omega_{1}{ }^{10}}-\frac{12555}{2048} \frac{c^{5} u_{1}{ }^{4} u_{2}{ }^{7}}{\omega_{1}{ }^{10}} \\
& +\frac{12555}{1024} \frac{c^{5} u_{1}{ }^{7} u_{2}{ }^{4}}{\omega_{1}{ }^{10}}+\frac{3}{16} \frac{c^{2} u_{1} u_{2}{ }^{4}}{\omega_{1}{ }^{4}} \\
& -\frac{97017}{65536} \frac{c^{5} u_{1}^{8} u_{2}^{3}}{\omega_{1}{ }^{10}}+\frac{759}{163840} \frac{c^{5} u_{1} u_{2}{ }^{10}}{\omega_{1}{ }^{10}} \\
& +\frac{915}{8192} \frac{c^{5} u_{1}{ }^{9} u_{2}{ }^{2}}{\omega_{1}{ }^{10}}-\frac{759}{131072} \frac{c^{5} u_{1}{ }^{10} u_{2}}{\omega_{1}{ }^{10}} \\
& -\frac{3}{4} \frac{c u_{1} u_{2}^{2}}{\omega_{1}{ }^{2}}-\frac{3}{8} \frac{c^{2} u_{1}^{4} u_{2}}{\omega_{1}{ }^{4}} \\
& -\frac{2745}{32768} \frac{c^{5} u_{1}^{2} u_{2}{ }^{9}}{\omega_{1}{ }^{10}}+\frac{51}{32} \frac{c^{2} u_{1}^{2} u_{2}^{3}}{\omega_{1}{ }^{4}} \\
& +\frac{32339}{32768} \frac{c^{5} u_{1}^{3} u_{2}^{8}}{\omega_{1}{ }^{10}}+\frac{3}{64} \frac{c^{2} u_{1}^{5}}{\omega_{1}{ }^{4}} \\
& +\frac{3}{16384} \frac{c^{5} u_{1}{ }^{11}}{\omega_{1}{ }^{10}}-\frac{5}{32768} \frac{c^{5} u_{2}{ }^{11}}{\omega_{1}{ }^{10}} \\
& -\frac{1}{32} \frac{c^{2} u_{2}^{5}}{\omega_{1}{ }^{4}}-\frac{1}{8} \frac{c u_{2}{ }^{3}}{\omega_{1}^{2}}+\frac{1}{4} \frac{c u_{1}^{3}}{\omega_{1}^{2}} \\
& -\frac{3}{512} \frac{c^{3} u_{2}{ }^{7}}{\omega_{1}{ }^{6}}+\frac{1}{128} \frac{c^{3} u_{1}{ }^{7}}{\omega_{1}{ }^{6}}-\frac{1}{1024} \frac{c^{4} u_{2}{ }^{9}}{\omega_{1}{ }^{8}} \\
& +\frac{5}{4096} \frac{c^{4} u_{1}{ }^{9}}{\omega_{1}{ }^{8}}-\frac{1773}{512} \frac{c^{4} u_{1}{ }^{6} u_{2}{ }^{3}}{\omega_{1}{ }^{8}} \\
& +\frac{843}{2048} \frac{c^{4} u_{1}^{7} u_{2}^{2}}{\omega_{1}^{8}}-\frac{59}{2048} \frac{c^{4} u_{1}^{8} u_{2}}{\omega_{1}^{8}}
\end{aligned}
$$

$$
\begin{aligned}
& +\frac{21}{256} \frac{c^{3} u_{1} u_{2}{ }^{6}}{\omega_{1}{ }^{6}}-\frac{273}{512} \frac{c^{3} u_{1}{ }^{2} u_{2}{ }^{5}}{\omega_{1}{ }^{6}} \\
& -\frac{147}{32} \frac{c^{3} u_{1}{ }^{3} u_{2}^{4}}{\omega_{1}{ }^{6}}+\frac{273}{256} \frac{c^{3} u_{1}{ }^{5} u_{2}{ }^{2}}{\omega_{1}{ }^{6}} \\
& -\frac{63}{512} \frac{c^{3} u_{1}{ }^{6} u_{2}}{\omega_{1}{ }^{6}}+\frac{177}{8192} \frac{c^{4} u_{1} u_{2}^{8}}{\omega_{1}^{8}} \\
& -\frac{281}{1024} \frac{c^{4} u_{1}{ }^{2} u_{2}{ }^{7}}{\omega_{1}^{8}}+\frac{1773}{1024} \frac{c^{4}}{u_{1}^{3} u_{2}{ }^{6}} \omega_{1}^{8} \\
& +\frac{31035}{2048} \frac{c^{4} u_{1}{ }^{4} u_{2} 5}{\omega_{1}{ }^{8}}
\end{aligned}
$$

with $\Phi_{2}$ conjugate of $\Phi_{1}$.

\section{Appendix B: Polynomials of Sect. 3.2}

Equation (27) corresponds to polynomial of degree at most 2 in $Q^{2}$.

If the degree is 1 , this is because:

$$
\begin{aligned}
& 3 s^{4} t^{2}+6 s^{2} t^{2}-9 / 4 r+3 t^{2}+3 r^{2}+\frac{15}{4} s^{4} r+9 s t \\
& +3 s^{3} t-9 / 2 r s^{2}-\frac{81}{64}+\frac{135}{32} s^{2}-\frac{225}{64} s^{4} \\
& +6 s^{2} r^{2}+3 s^{4} r^{2}=0 .
\end{aligned}
$$

But this is impossible for any $s$ since for $s=0, s=1$, $s=-1$, we obtain

$$
\begin{aligned}
& -\frac{81}{64}-9 / 4 r+3 t^{2}+3 r^{2}=0 \\
& 12 t^{2}-3 r+12 r^{2}+12 t-\frac{9}{16}=0,
\end{aligned}
$$

and

$12 t^{2}-3 r+12 r^{2}-12 t-\frac{9}{16}=0$.

These equations have no common solutions. So, we can consider the general case with degree 2 . We examine the condition so that the polynomial of variable $Q^{2}$ (Eq. (27)) has no real solutions for any $s$. So we demand that the discriminant that is equal to

$$
\begin{aligned}
& \left(8 s^{2}+4 s^{4}+4\right) r^{2}+\left(9+18 s^{2}-15 s^{4}\right) r+\frac{225}{16} s^{4} \\
& -12 s^{3} t-12 t^{2}-12 s^{4} t^{2}-24 s^{2} t^{2}+\frac{81}{16}
\end{aligned}
$$




$$
\begin{aligned}
& -36 s t-\frac{135}{8} s^{2}+\frac{225}{16} s^{4}-12 s^{3} t-12 t^{2} \\
& -12 s^{4} t^{2}-24 s^{2} t^{2}+\frac{81}{16}-36 s t-\frac{135}{8} s^{2}
\end{aligned}
$$

should be $<0$ for any $s$. This discriminant is a polynomial of degree 2 in $r$ that should have a constant sign. Let us examine the discriminant of this later polynomial in $r$. It is equal to

$$
\begin{aligned}
- & 4\left(8 s^{2}+4 s^{4}+4\right)\left(-24 s^{2}-12-12 s^{4}\right) t^{2} \\
- & 4\left(8 s^{2}+4 s^{4}+4\right)\left(-36 s-12 s^{3}\right) t \\
+ & \left(9+18 s^{2}-15 s^{4}\right)^{2}-4\left(8 s^{2}+4 s^{4}+4\right) \\
& \times\left(\frac{225}{16} s^{4}+\frac{81}{16}-\frac{135}{8} s^{2}\right)
\end{aligned}
$$

It is clear that either the polynomial changes the sign or it is a constant sign but positive. As a conclusion, it is impossible to suppress the occurrence of singularities in the normal transform calculation up to order 3 by the choice of a nonzero arbitrary $\beta$.

\section{References}

1. Poincaré, H.: Les Méthodes Nouvelles de la Mécanique Céleste. Gauthiers-Villars, Paris (1892)

2. Sanders, J.A., Verhulst, F., Murdock, S.: Averaging Methods in Nonlinear Dynamical Systems, 2nd edn. Springer, Berlin (2007)

3. Gutzwiller, M.C.: Moon-Earth-Sun: the oldest three-body problem. Rev. Mod. Phys. 70, 589-639 (1998)

4. Nayfeh, A.H., Mook, D.T.: Nonlinear Oscillations. Wiley, New York (1979)

5. Nayfeh, A.H.: Nonlinear Interactions: Analytical, Computational and Experimental Methods. Wiley Series in Nonlinear Science. Wiley, New York (2000)

6. Nayfeh, A.H.: Method of Normal Forms. Wiley, New York (1993)

7. Dulac, H.: Solutions d'un système d'équations différentielles dans le voisinage de valeurs singulières. Bull. Soc. Math. Fr. 40, 324-383 (1912)

8. Iooss, G., Adelmeyer, M.: Topics in Bifurcation Theory and Applications. World Scientific, Singapore (1992)

9. Elphick, C., Tirapegui, E., Brachet, M.E., Coullet, P., Iooss, G.: A simple global characterization for normal forms of singular vector fields. Physica D 29, 95-127 (1987)

10. Iooss, G.: Global characterization of the normal form for a vector field near a closed orbit. J. Differ. Equ. 76, 47-76 (1988)
11. Guckenheimer, J., Holmes, P.: Nonlinear Oscillations, Dynamical Systems and Bifurcations of Vector Fields. Springer, New York (1983)

12. Carr, J.: Applications of Center Manifold Theory. Applied Mathematical Sciences, vol. 35. Springer, New York (1981)

13. Mielke, A.: Hamiltonian and Lagrangian Flows on Center Manifolds: With Applications to Elliptic Variational Problems. Lecture Notes in Mathematics, vol. 1489. Springer, Berlin (1991)

14. Jézéquel, L., Lamarque, C.-H.: Analysis of nonlinear structural vibrations by normal form theory. J. Sound Vib. 149(3), 429-459 (1991)

15. Lamarque, C.-H.: Contribution à la Modélisation et à l'identification des Systèmes Mécaniques Non-Linéaires, Thèse de Doctorat de 1'Ecole Centrale de Lyon, Spécialité Mécanique, numéro d'ordre 92-32 (1992)

16. Brjuno, A.D.: Analytical forms of differential equations. Tr. Mosk. Mat. Obŝ. 25, 119-262 (1971)

17. Brjuno, A.D.: Analytical forms of differential equations. Tr. Mosk. Mat. Obŝ. 25, 199-299 (1972)

18. Yu, P.: Computation of normal forms via a perturbation technique. J. Sound Vib. 211(1), 19-38 (1998)

19. Zhang, W., Huseyin, K.: On the computation of the coefficients associated with high order normal forms. J. Sound Vib. 232(3), 525-540 (2000)

20. Neild, S.A., Wagg, D.J.: Applying the method of normal forms to second-order nonlinear vibration problem. Proc. R. Soc. A, Math. Phys. Eng. Sci. 467, 1141-1163 (2011)

21. Coullet, P.H., Spiegel, E.A.: Amplitude equation for systems with competing instabilities. SIAM J. Appl. Math. 43(4), 776-821 (1985)

22. Marsden, J.E., Mc Cracken, M.: The Hopf Bifurcation and Its Applications. Applied Mathematical Sciences, vol. 19. Springer, New York Heidelberg Berlin (1976).

23. Golubitsky, M.S., Schaeffer, D.: Singularities and Groups in Bifurcation Theory, vol. I. Appl. Math. Sci., vol. 51. Springer, New York (1985)

24. Golubitsky, M.S., Stewart, I., Schaeffer, D.: Singularities and Groups in Bifurcation Theory, vol. II. Appl. Math. Sci., vol. 69. Springer, New York (1988)

25. Touzé, C., Thomas, O., Chaigne, A.: Hardening/softening behaviour in non-linear oscillations of structural systems using non-linear normal modes. J. Sound Vib. 273(1-2), 77-101 (2004)

26. Touzé, C., Amabili, M.: Non-linear normal modes for damped geometrically non-linear systems: application to reduced-order modeling of harmonically forced structures. J. Sound Vib. 298(4-5), 958-981 (2006)

27. Amabili, M., Touzé, C.: Reduced-order models for nonlinear vibrations of fluid-filled circular cylindrical shells: comparison of POD and asymptotic non-linear normal modes methods. J. Fluids Struct. 23(6), 885-903 (2007)

28. Touzé, C., Amabili, M., Thomas, O.: Reduced-order models for large-amplitude vibrations of shells including inplane inertia. Comput. Methods Appl. Mech. Eng. 197(2124), 2030-2045 (2008)

29. Karkar, S., Cochelin, B., Vergez, C., Thomas, O., Lazarus, A.: USER GUIDE MANLAB $\mathbf{2 . 0}$ (2010). http://manlab.lma.cnrs-mrs.fr/ 
30. Cochelin, B., Vergez, C.: A high order purely-based harmonic balance formulation for continuation of periodic solutions. J. Sound Vib. 324, 243-262 (2009)

31. Lazarus, A., Thomas, O.: A harmonic-based method for computing the stability of periodic solutions of dynamical systems. C. R., Méc. 338, 510-517 (2010)

32. Shaw, S.W., Pierre, C.: Non-linear normal modes and invariant manifolds. J. Sound Vib. 150(1), 170-173 (1991)
33. Shaw, S.W., Pierre, C.: Normal modes for non-linear vibratory systems. J. Sound Vib. 164(1), 85-124 (1993)

34. Pellicano, F., Mastroddi, F.: Applicability conditions of a non-linear superposition technique. J. Sound Vib. 200(1), 3-14 (1997) 